\title{
49. GEOCHEMISTRY AND ORIGIN OF EAST PACIFIC SEDIMENTS SAMPLED DURING DSDP LEG 34
}

\author{
Kurt Boström,' Oiva Joensuu, Sylvia Valdés, Wally Charm, and Robert Glaccum, \\ Rosenstiel School of Marine and Atmospheric Science, University of Miami, Miami, Florida
}

\section{INTRODUCTION}

Several papers have recently appeared about the metalliferous sediments on the active (spreading) oceanic ridges. The geochemistry and mineralogy of such sediments are now reasonably well known, and generally these deposits are considered to be products of volcanic processes. The nature of this volcanism is still unclear: both deep-seated volcanic centers (Arrhenius and Bonatti, 1965; Boström and Peterson, 1966) and shallow hydrothermal leaching of basalts (Boström, 1967; Corliss, 1971) have been discussed as possible sources for the metal-rich sediments. However, such hydrothermal leaching is probably an insufficient process since it neither accounts for the observed accumulation rates for iron and manganese, nor for the observed $\mathrm{Fe} / \mathrm{Al}$ and $\mathrm{Fe} / \mathrm{Ti}$ ratios in the sediments (Boström, 1973; in press). Biological sources possibly account for some traces, (e.g., $\mathrm{Ba}$ and $\mathrm{Cu}$, Turekian, 1968; Boström et al., 1974b; Moore et al., in preparation), but to what extent is not known. The need for further studies of active ridge deposits is therefore great, particularly in view of the significance such deposits may have as sources for primary or secondary enrichment of $\mathrm{Fe}, \mathrm{Mn}, \mathrm{Cu}$, etc. in various economic deposits (Sillitoe, 1972; Boström et al., 1972b; Boström, 1973). The sampling by Glomar Challenger in an area with well-developed active ridge deposits was therefore of utmost importance for a discussion of how active ridge deposits originate.

\section{EXPERIMENTAL METHODS AND RESULTS}

The material for this study (about 150 samples) was collected by one of us (Charm) on board Glomar Challenger. The procurement of material immediately after the recovery of the sediment cores considerably decreased the risk of contamination, which may affect samples after long storage. The samples were never taken close to the core liner or the surface of splitting in order to lessen the risk for contamination. The strong resemblance between the analytical results presented here and in many other publications (Boström et al., 1972b; Boström, 1973) suggests that the contamination is negligible in most studies in the past as well as in this study.

After being transferred to the University of Miami, 95 samples were selected for analysis, which were dried, ground, and subsequently split into fractions. One fraction was analyzed by atomic absorption spectrophotometry (S.V.) for $\mathrm{NaCl}, \mathrm{CaCO}_{3}, \mathrm{Si}, \mathrm{Al}, \mathrm{Fe}, \mathrm{Mn}, \mathrm{P}$,

'Present address: Department of Economic Geology, University of Lulea, 95187 , Sweden.
$\mathrm{Cu}, \mathrm{Zn}, \mathrm{Mg}$, and $\mathrm{Sr}$ (of which the two last elements are not further discussed in this paper). Another fraction was analyzed by emission spectrometry (O.J.) for $\mathrm{Si}$, Ti, $\mathrm{Fe}, \mathrm{Mn}, \mathrm{P}, \mathrm{B}, \mathrm{Ba}, \mathrm{Cu}, \mathrm{Co}, \mathrm{Ni}, \mathrm{La}, \mathrm{Sc}, \mathrm{Y}, \mathrm{Cr}, \mathrm{V}$, and $\mathrm{Zr}$. Procedures and analytical accuracies are similar to those reported in Boström and Peterson, 1969; Boström et al., 1972b.

The distribution of the samples and the original values are given in Tables 1 and 2 . Table 3 presents a grouping of the material into chronological units, for which accumulation rates can be calculated. Tables 4 to 8 and Figures 1 to 4 present additional material that will be discussed in the following. In this paper the chemistry of concretionary matter, oxide coatings on rocks, etc. have been disregarded, since their total masses and rates of accumulation are small compared to other deep-sea sediments.

\section{SEDIMENT-PETROGRAPHIC NORM CALCULATIONS}

Norm calculations have played a large role in the study of igneous rocks, but have been used infrequently by sedimentologists. The motivation for norm calculations is that "rock analyses can be quantitatively studied and compared from a petrological point of view" (Barth, 1952).

In this paper, extensive use is made of such calculations. As was pointed out in Boström et al. (1972b), recalculation on a carbonate-free basis is a poor procedure, particularly when the carbonate content is high; then even small fluctuations (that is, errors) in $\mathrm{CaCO}_{3}$ content will lead to sharp fluctuations in the carbonate-free basis values. This can be corrected if very careful analyses are produced for the carbonate, total interstitial salt, organic matter, and total water content. Unfortunately, this procedure involves much work and is expensive and time consuming. A simpler procedure is to recalculate the sum of the major oxides, sulfates, etc., to a given sum as is outlined in the note for Table 5; by this method even the diluting effect by opaline silica can be removed.

We do not claim that these procedures represent the final end product; certainly many improvements can be added. Yet these norms, as well as the element ratios presented in Figures 1, 2, and 3 do give a clearer picture of the compositional relationships in deep-sea sediments than do the unprocessed data in Tables 2 or 4.

\section{DISCUSSION}

\section{Chemical Analyses}

The results reported in Table 2 show striking similarities with data already published elsewhere. As in 
TABLE 1

Sample Distribution

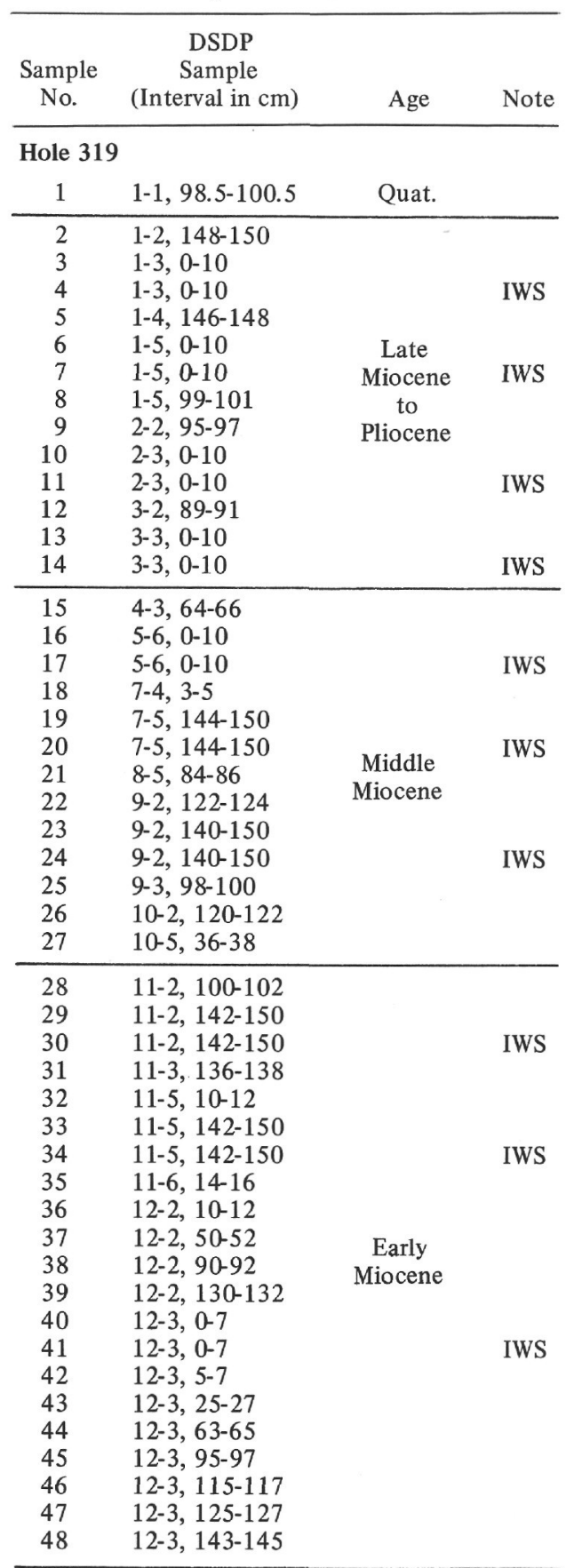

Hole 320

\begin{tabular}{llrl}
49 & $1-1,20-22$ & & \\
50 & $1-1,36-39$ & & \\
51 & $1-1,62-64$ & & \\
52 & $1-2,68-70$ & Quat. & \\
53 & $1-4,57-59$ & & \\
54 & $1-6,71-73$ & & IWS \\
55 & $1-2,144-150$ & & IWS \\
56 & $1-5,144-150$ & & \\
\hline 57 & $2-2,99-101$ & & \\
58 & $3-2,136-138$ & Early & \\
59 & $3-2,144-150$ & Miocene & \\
60 & $3-4,74-76$ & & \\
61 & $3-6,122-124$ & & \\
\hline
\end{tabular}

TABLE 1 - Continued

\begin{tabular}{|c|c|c|c|}
\hline $\begin{array}{l}\text { Sample } \\
\text { No. }\end{array}$ & $\begin{array}{c}\text { DSDP } \\
\text { Sample } \\
\text { (Interval in } \mathrm{cm} \text { ) }\end{array}$ & Age & Note \\
\hline \multicolumn{4}{|c|}{ Hole 320B } \\
\hline $\begin{array}{l}62 \\
63 \\
64 \\
65 \\
66 \\
67 \\
68 \\
69\end{array}$ & $\begin{array}{l}1-1,129-131 \\
1-3,138-140 \\
1-5,122-124 \\
1-5,143-150 \\
2-1,86-88 \\
2-3,111-113 \\
2-5,132-134 \\
2-5,140-150\end{array}$ & $\begin{array}{c}\text { Late } \\
\text { Oligocene } \\
\text { to } \\
\text { early } \\
\text { Miocene }\end{array}$ & IWS \\
\hline \multicolumn{4}{|l|}{ Site 321} \\
\hline $\begin{array}{l}70 \\
71 \\
72 \\
73 \\
74\end{array}$ & $\begin{array}{l}2-2,56-58 \\
2-4,0-6 \\
3-2,101-102 \\
4-2,56-58 \\
4-3,144-150\end{array}$ & Quat. & $\begin{array}{l}\text { IWS } \\
\text { IWS }\end{array}$ \\
\hline 75 & $5-4,138-140$ & Pliocene-Ple & stocene \\
\hline $\begin{array}{l}76 \\
77 \\
78 \\
79 \\
\end{array}$ & $\begin{array}{l}6-2,106-108 \\
6-4,130-132 \\
6-4,144-150 \\
6-6,142-144 \\
\end{array}$ & $\begin{array}{c}\text { Late } \\
\text { Miocene }\end{array}$ & IWS \\
\hline $\begin{array}{l}80 \\
81 \\
82 \\
83 \\
84 \\
85 \\
86\end{array}$ & $\begin{array}{l}7-2,138-140 \\
7-4,139-141 \\
7-6,125-127 \\
8-1,135-137 \\
9-1,15-17 \\
9-5,31-33 \\
9-6,0-6\end{array}$ & $\begin{array}{c}\text { Late } \\
\text { Oligocene } \\
\text { to } \\
\text { early } \\
\text { Miocene }\end{array}$ & IWS \\
\hline 87 & $10-2,120-122$ & Middle Olig & cene \\
\hline $\begin{array}{l}88 \\
89 \\
\end{array}$ & $\begin{array}{l}11-2,90-92 \\
11-4,0-6\end{array}$ & $\begin{array}{c}\text { Early } \\
\text { Oligocene }\end{array}$ & IWS \\
\hline $\begin{array}{l}90 \\
91 \\
92 \\
93 \\
94 \\
95\end{array}$ & $\begin{array}{l}13-1,145-147 \\
13-3,0-6 \\
13-3,42-44 \\
13-3,82-84 \\
13-3,102-104 \\
13-3,122-124\end{array}$ & $\begin{array}{l}\text { Late } \\
\text { Eocene }\end{array}$ & IWS \\
\hline
\end{tabular}

the DSDP crossing in the South Atlantic (Leg 3, Boström et al. 1792b), there is a pronounced tendency for the Fe-, Mn-, V-rich and Si-, Al-, Ti-poor sediments to occur in the basal layer of the sediments. Thus, all sediments that are rich in $\mathrm{Fe}, \mathrm{Mn}, \mathrm{P}_{2} \mathrm{O}_{5}$, and $\mathrm{V}$, or show high accumulation rates for these constituents, were formed within $20 \mathrm{~m} . \mathrm{y}$. from the generation of their basaltic substratum (groups A, B, C, D, F, G, K, L, M, and $N$ ). This is the distribution pattern one would expect if ferromanganoan sediments are the first to form at spreading centers (Boström and Peterson, 1969) and confirms the validity of this interpretation. There is now little doubt that such sediments exclusively originate on active ridges, partly involving some form of submarine volcanism. The data in Figures 1 and 2 suggest that the volcanic source delivers material of remarkably constant composition; otherwise, a much larger scatter should be expected in the petrochemical graphs. The same graphs also clearly demonstrate that the only other phase of 
significance that involves $\mathrm{Fe}, \mathrm{Mn}, \mathrm{Al}$, and $\mathrm{Ti}$ is terrigenous material (TM as defined in Table 8); most remarkable is the negligible significance of lithogenic matter of basaltic origin. (See also Boström et al., 1973b.)

\section{Accumulation Rates}

The data in Tables 3 and 4 have been used to calculate the accumulation rates presented in Table 6 . The data show that the highest accumulation rates of authigenic Fe-Mn phases occur close to spreading centers, that is, in the basal sediments. Similar patterns are found for B, $\mathrm{Cu}, \mathrm{Ni}, \mathrm{V}, \mathrm{P}$, and $\mathrm{Zn}$ whereas the pattern is opposite for $\mathrm{Al}$ and $\mathrm{Ti}$. Less clear are the patterns for $\mathrm{Co}, \mathrm{Y}, \mathrm{Zr}$, $\mathrm{SiO}_{2}$ *, and $\mathrm{Ba}$ (for which nevertheless some of the high $\mathrm{Ba}$ accumulation rates are found in sediments with high accumulation rates for $\mathrm{Fe}$ and $\mathrm{Mn}$ ). There are, however, problems with these calculated accumulation rates. Uncertainties in dating and in physical properties of the sediments may give errors on the order of $10 \%-20 \%$ in the values presented. More serious are the errors that may be physiographically induced; the topography of the ocean floor may enhance or lessen the observed accumulation rates due to slumping, turbidite flows, or gentle winnowing of matter (van Andel et al., 1967). Bottom currents obviously intensify the disturbing processes. As a result of such processes, undisturbed cores suitable for dating and accumulation rate studies are difficult to obtain on the Mid-Atlantic Ridge (MAR). The sediment distribution on the East Pacific Rise (EPR), on the other hand, is different. Thus, the EPR is characterized by a very smooth and gentle topography and only close to the spreading center does bare rock make up considerably more than $50 \%$ of the area (Boström et al., 1974a). Because of this fact, extensive coring was performed on or close to the crest of the EPR at about $14^{\circ}-15^{\circ} \mathrm{S}$ and several 8-9 meter long sediment cores were procured, even on topographic elevations during cruise GS 7202 of the University of Miami (Spring 1972). Most remarkable was an 8.5-meter long core (GS 7202-35 P) recovered from a hilltop only $80 \mathrm{~km}$ west of the spreading center. Studies of this core suggest essentially undisturbed sedimentation for at least the last 600,000 years; the only disturbance of any significance may have been a slight loss of fines from the hilltop due to down-slope winnowing (Boström et al., 1974a). The accumulation rates for core GS7202-35 P presented in Table 6 may therefore be too how, but show good orderof-magnitude agreement with accumulation rates for groups $\mathrm{C}$ and $\mathrm{D}$.

The accumulation rates for the sediments at Site 319 may nevertheless be somewhat high. Studies of PDR records at University of Miami and at Scripps Institution of Oceanography, as well as coring results, suggest that about $70 \%$ of the present Bauer Deep is sediment covered (Boström et al., 1973a) and airgun profiles suggest that the older sediments cover about $30 \%-35 \%$. This implies that observed accumulation rates, at least for the lower part of Hole 319, may be about twice as large as undisturbed, evenly distributed accumulation would give. Similar comments may pertain to the lowest parts of Hole 320 and Site 321 . However, the difficulties in correctly assessing the influence of the "paleotopography" when the basal sediments were deposited at Hole 320 and Site 321 makes it difficult to know with certainty whether the accumulation rates in the basal layers may be too low or too high. Nevertheless, the remarkable similarities in chemistry and overall accumulation rates for many constituents in the basal sediments in Holes 319-321 with recently forming deposits on the EPR strongly suggest that the rapid formation of Fe-Mn-rich, Al-Ti-poor sediments has been a nearly continuous process at the East Pacific spreading centers (either on the EPR or on the Galapagos Rise) for the last $36 \mathrm{~m} . \mathrm{y}$. This is particularly well emphasized with the normalized values presented in Table 5.

\section{Relationships Between Spreading Rates and the Chemistry of the Sediments}

Previous studies show that there is a distinct relationship between the composition of basal sediments and the spreading rates at the plate-generating zone, (see Figure 3 and Boström, 1973).

The relationship $(\mathrm{Fe}+\mathrm{Mn}) / \mathrm{Al}$ can be written as an exponential function of the spreading rate as follows:

$$
\frac{\mathrm{Fe}+\mathrm{Mn}}{\mathrm{Al}}=0.59 \times e^{0.53 \mathrm{SR}}
$$

where $\mathrm{SR}=$ spreading rate in $\mathrm{cm} /$ year.

This relationship indicates that exhalations at centers with rapid spreading surface rapidly implying that the rate of admixture of terrigenous, basaltic, and biogenic matter must be outpaced, which accounts for the variations in the ratio $(\mathrm{Fe}+\mathrm{Mn}) / \mathrm{Al}$. Such variations cannot be deduced from the data in Figure 1 alone. This spreading-rate dependence for $\mathrm{Fe}$ and $\mathrm{Mn}$ strongly suggests an intimate connection with those volcanic processes that are associated with the rate of generation of new basaltic crust, that is, possibly with the splitting of pyrolite into residual peridotite, basalt, and a minor quantity of exhalations. For the basal sediments from Holes 319-321 this $(\mathrm{Fe}+\mathrm{Mn}) / \mathrm{Al}$ ratio varies between 15 and 23. According to the best-fit curve, this would correspond to a spreading rate of $6.0-6.7 \mathrm{~cm} /$ year (see Figure 3 ), which is very close to the spreading rates of 6$7 \mathrm{~cm} /$ year for those sites where Holes 319, 320, and 321 are located. Hole 319 is associated with a segment of the EPR that has spreading rates of about $6-7 \mathrm{~cm} /$ year according to Herron (1972). Hole 320 and Site 321 are located approximately $1700 \mathrm{~km}$ from the old Galapagos spreading center and were displaced by spreading from about 35 m.y. B.P. (age of the basal sediment) until 10 m.y. B.P., at which time the Galapagos Ridge ceased to be active (Hays et al., 1972). This implies that spreading took place at a little less than $7 \mathrm{~cm} /$ year.

\section{Origin of Major Constituents in the Basal Sediments}

The well-defined main element composition of the active ridge-derived matter (see Figures 1 and 2) and the relationship between spreading rates and $(\mathrm{Fe}+\mathrm{Mn}) / \mathrm{Al}$ values point to an origin related to the same process that controls the spreading rate. A possible interpretation is 
TABLE 2

Original Analytical Results for Sediment Samples Obtained During DSDP Leg 34

\begin{tabular}{|c|c|c|c|c|c|c|c|c|c|c|c|c|c|c|c|c|c|c|c|}
\hline $\begin{array}{l}\text { Sample } \\
\text { No. }\end{array}$ & $\begin{array}{l}\mathrm{Na} \\
(\%)\end{array}$ & $\begin{array}{l}\mathrm{SiO}_{2} \\
(\%)\end{array}$ & $\begin{array}{l}\mathrm{Ti} \\
(\%)\end{array}$ & $\begin{array}{l}\mathrm{Al} \\
(\%)\end{array}$ & $\begin{array}{l}\mathrm{Fe} \\
(\%)\end{array}$ & $\begin{array}{l}\mathrm{Mn} \\
(\%)\end{array}$ & $\begin{array}{c}\mathrm{P}_{2} \mathrm{O}_{5} \\
(\%)\end{array}$ & $\begin{array}{c}\mathrm{B} \\
(\mathrm{ppm})\end{array}$ & $\begin{array}{c}\mathrm{Ba} \\
(\mathrm{ppm})\end{array}$ & $\begin{array}{c}\mathrm{Co} \\
(\mathrm{ppm})\end{array}$ & $\begin{array}{c}\mathrm{Cr} \\
(\mathrm{ppm})\end{array}$ & $\begin{array}{c}\mathrm{Cu} \\
\text { (ppm) }\end{array}$ & $\begin{array}{c}\mathrm{La} \\
(\mathrm{ppm})\end{array}$ & $\begin{array}{c}\mathrm{Ni} \\
(\mathrm{ppm})\end{array}$ & $\underset{(\mathrm{ppm})}{\mathrm{Sc}}$ & $\begin{array}{c}\mathrm{V} \\
(\mathrm{ppm})\end{array}$ & $\begin{array}{c}\mathrm{Y} \\
(\mathrm{ppm})\end{array}$ & $\begin{array}{c}\mathrm{Zr} \\
(\mathrm{ppm})\end{array}$ & $\underset{(\mathrm{ppm})}{\mathrm{Zn}}$ \\
\hline 1 & 5.3 & 29 & 0.16 & 2.5 & 13.3 & 3.99 & 2.1 & 150 & 9100 & 220 & 13 & 850 & 280 & 540 & 17 & 190 & 1200 & 720 & 795 \\
\hline 2 & 4.7 & 29 & 0.32 & 2.2 & 14.1 & 3.30 & 1.7 & 170 & 9700 & 180 & 8 & 890 & 230 & 540 & 16 & 220 & 620 & 310 & 340 \\
\hline 3,4 & 4.7 & 30.5 & 0.24 & 2.4 & 14.6 & 3.45 & 1.8 & 165 & 12500 & 175 & 11 & 910 & 200 & 540 & 14 & 230 & 555 & 240 & 347 \\
\hline 5 & 3.1 & 18 & 0.090 & 2.3 & 14.7 & 3.57 & 1.5 & 160 & 8000 & 140 & 10 & 890 & 190 & 360 & 8.5 & 270 & 360 & 250 & 330 \\
\hline 6,7 & 1.7 & 17.2 & 0.076 & 1.2 & 15.6 & 3.54 & 1.4 & 130 & 7400 & 135 & 6 & 880 & 150 & 250 & 8.2 & 290 & 290 & 210 & 325 \\
\hline 8 & 0.89 & 11.7 & 0.030 & 0.47 & 6.3 & 1.47 & 0.61 & 60 & 3900 & 52 & $<10$ & 390 & 35 & 140 & $\mathrm{NA}$ & 130 & 120 & 70 & 175 \\
\hline 9 & 0.45 & 12.0 & 0.022 & 0.25 & 5.7 & 0.89 & 0.32 & 45 & 3300 & 24 & $<10$ & 320 & 9 & 90 & NA & 60 & 60 & 48 & 120 \\
\hline 10,11 & 0.85 & 11.6 & 0.024 & 0.28 & 5.1 & 1.09 & 0.29 & 46 & 3000 & 22 & $\sim 2$ & 330 & 27 & 85 & $\sim 2.0$ & 75 & 57 & 52 & 175 \\
\hline 12 & 0.82 & 14.3 & NA & 0.38 & 6.9 & 1.24 & 0.45 & NA & NA & NA & NA & NA & NA & NA & NA & NA & $\mathrm{NA}$ & NA & NA \\
\hline 13,14 & 1.4 & 16.8 & 0.028 & 0.47 & 7.8 & 1.55 & 0.55 & 72 & 3600 & 19 & & 510 & 45 & 115 & 3.5 & 135 & 115 & 100 & 180 \\
\hline 15 & 0.82 & 2.3 & 0.0070 & 0.16 & 2.7 & 0.56 & 0.25 & 50 & 1200 & 7 & $<5$ & 160 & 42 & 42 & $<2$ & 85 & 42 & 32 & 145 \\
\hline 16,17 & 0.50 & 0.63 & 0.0015 & 0.040 & 0.67 & 0.15 & 0.12 & 30 & 360 & 5 & $<5$ & 57 & $<10$ & 10 & $<2$ & 10 & 12 & 9 & 43 \\
\hline 18 & 0.74 & 1.3 & 0.0030 & 0.11 & 1.2 & 0.29 & 0.16 & 37 & 720 & 7 & $<5$ & 94 & $<10$ & 15 & 4 & 23 & 22 & 16 & 160 \\
\hline 19,20 & 0.60 & 1.0 & 0.0022 & 0.075 & 0.85 & 0.21 & 0.12 & 48 & 780 & 5.7 & $<5$ & 68 & $<10$ & 15 & 4 & 17 & 16 & 11 & 42 \\
\hline 21 & 0.78 & 1.9 & 0.013 & 0.15 & 2.0 & 0.56 & 0.25 & 64 & NA & 19 & $<5$ & 180 & 25 & 42 & 8 & 90 & 36 & 32 & 77 \\
\hline 22 & 0.80 & 2.8 & 0.016 & 0.23 & 5.0 & 2.17 & 0.37 & 70 & 1050 & 27 & $<$ & 350 & 33 & 150 & 7 & 210 & 42 & 45 & 170 \\
\hline 23,24 & 0.66 & 1.7 & 0.010 & 0.15 & 2.9 & 1.80 & 0.30 & 50 & 1350 & 20 & $<5$ & 200 & 21 & 100 & $<2$ & 125 & 28 & 28 & 100 \\
\hline 25 & 1.26 & 3.6 & 0.019 & 0.29 & 5.2 & 2.55 & 0.43 & 70 & 1020 & 32 & $<5$ & 430 & 33 & 200 & 2.5 & 230 & 44 & 48 & 210 \\
\hline 26 & 0.74 & 1.1 & 0.0078 & 0.080 & 2.0 & 0.60 & 0.20 & 45 & 560 & 18 & $<5$ & 120 & $<10$ & 60 & $<2$ & 75 & 22 & 22 & 70 \\
\hline 27 & 0.74 & 1.0 & 0.0084 & 0.11 & 1.9 & 0.62 & 0.18 & 30 & 720 & 19 & $<5$ & 135 & $<10$ & 60 & $<2$ & 75 & 16 & 20 & 83 \\
\hline 28 & 0.73 & 1.2 & 0.0096 & 0.080 & 2.4 & 1.00 & 0.25 & 42 & 660 & 42 & $<5$ & 165 & $<10$ & 90 & $<2$ & 100 & 26 & 26 & 96 \\
\hline 29,30 & 0.56 & 1.0 & 0.0069 & 0.095 & 1.9 & 0.70 & 0.24 & 39 & 515 & 8 & $<$ & 125 & NA & 67 & $\sim 2$ & 80 & 16 & 17 & 80 \\
\hline 31 & 0.91 & 2.7 & 0.017 & 0.21 & 5.2 & 2.17 & 0.40 & 65 & 1100 & 30 & $<5$ & 320 & 30 & 180 & 6.5 & 190 & 30 & 40 & 175 \\
\hline 32 & 0.49 & 1.6 & 0.0078 & 0.13 & 3.0 & 0.17 & 0.27 & 50 & 620 & 18 & $<5$ & 150 & 12 & 70 & 3.5 & 85 & 16 & 20 & 85 \\
\hline 33,34 & 0.64 & 1.4 & 0.0078 & 0.12 & 2.2 & 0.70 & 0.24 & 47 & 810 & 14 & $<$ & 130 & $\sim 6$ & 40 & 3.0 & 64 & 16 & 16 & 70 \\
\hline 35 & 0.70 & 2.1 & 0.0102 & 0.16 & 4.4 & 1.29 & 0.34 & 60 & 1100 & 17 & $<$ & 155 & 13 & 110 & 3.5 & 95 & 21 & 22 & 110 \\
\hline 36 & 0.62 & 1.9 & 0.0084 & 0.16 & 2.2 & 0.62 & 0.27 & 40 & 380 & 18 & $<$ & 135 & 14 & 53 & 4.5 & 60 & 16 & 20 & 69 \\
\hline 37 & 0.61 & 1.7 & 0.0078 & 0.15 & 1.5 & 0.41 & 0.25 & 37 & 830 & 11 & $<$ & 80 & $<10$ & 35 & $<2$ & 30 & 30 & 13 & 39 \\
\hline 38 & 0.59 & 1.6 & 0.0072 & 0.16 & 1.2 & 0.33 & 0.25 & 35 & 820 & 8 & $<$ & 85 & $<10$ & 19 & $<2$ & 24 & 24 & 13 & 62 \\
\hline 39 & 0.55 & 1.6 & 0.0066 & 0.11 & 0.85 & 0.21 & 0.22 & 45 & 850 & 7 & $<$ & 55 & $<10$ & 17 & $<2$ & 16 & 16 & 13 & 41 \\
\hline 40,41 & 0.37 & 1.2 & 0.0063 & 0.11 & 0.93 & 0.23 & 0.20 & 32 & 760 & 7 & $<$ & 55 & $<10$ & 17 & $<2$ & 15 & 12 & 12 & 37 \\
\hline 42 & 0.58 & 1.3 & 0.0072 & 0.11 & 1.0 & 0.23 & 0.21 & 35 & 710 & 11 & $<$ & 55 & $<10$ & 20 & $<2$ & 18 & 12 & 13 & 42 \\
\hline 43 & 0.50 & 1.1 & 0.0060 & 0.080 & 0.75 & 0.19 & 0.21 & 35 & 730 & 6.5 & $<$ & 45 & $<10$ & 17 & $<2$ & 17 & 12 & 12 & 41 \\
\hline 44 & 0.52 & 1.3 & 0.0072 & 0.10 & 0.95 & 0.25 & 0.24 & 55 & 650 & 14 & $<$ & 60 & $<10$ & 21 & $<2$ & 22 & 19 & 25 & 42 \\
\hline 45 & 0.86 & 6.0 & 0.021 & 0.28 & 4.2 & 1.11 & 0.33 & 65 & 1450 & 16 & $<$ & 85 & $<10$ & 95 & $<2$ & 90 & 24 & 40 & 120 \\
\hline 46 & 0.56 & 1.7 & 0.016 & 0.13 & 1.5 & 0.40 & 0.28 & 37 & 720 & 14 & $<$ & 120 & $<10$ & 37 & $<2$ & 41 & 16 & 17 & 58 \\
\hline 47 & 0.65 & 2.4 & 0.013 & 0.21 & 2.6 & 0.88 & 0.32 & 70 & 780 & 19 & $<$ & 135 & $<10$ & 27 & $<2$ & 75 & 22 & 20 & 81 \\
\hline 48 & 0.59 & 3.4 & 0.0078 & 0.13 & 2.7 & 0.61 & 0.31 & 40 & 1200 & 12 & $<5$ & 140 & $<10$ & 50 & $<2$ & 55 & 15 & 17 & 70 \\
\hline 49 & 3.12 & 54 & 0.34 & 10.21 & 4.3 & 0.081 & 0.14 & 130 & 3300 & 17 & 95 & 190 & 25 & 160 & 16 & 195 & 22 & 122 & 330 \\
\hline 50 & 3.71 & 48 & 0.34 & 10.21 & 4.2 & 0.620 & 0.18 & 95 & 3900 & 20 & 75 & 290 & 28 & 180 & 14 & 190 & 26 & 122 & 250 \\
\hline 51 & 3.71 & 52 & 0.38 & 7.94 & 4.3 & 0.081 & 0.14 & 110 & 2700 & 22 & 86 & 240 & 30 & 240 & 18 & 270 & 22 & 120 & 330 \\
\hline 52 & 4.10 & 52 & 0.37 & 5.93 & 3.4 & 0.121 & 0.12 & 105 & 2900 & 14 & 47 & 360 & 30 & 240 & 14 & 220 & 20 & 100 & 390 \\
\hline 53 & 3.49 & 50 & 0.32 & 6.46 & 5.8 & 0.089 & 0.11 & 115 & 3100 & 24 & 59 & 220 & 25 & 320 & 14 & 200 & 17 & 110 & 250 \\
\hline 54 & 3.26 & 50 & 0.32 & 7.41 & 4.2 & 0.124 & 0.21 & 115 & 3300 & 14 & 95 & 240 & 28 & 190 & 14 & 165 & 31 & 120 & 310 \\
\hline 55 & 2.60 & 53 & 0.39 & 8.2 & 4.4 & 0.074 & 0.18 & 110 & 4400 & 13 & 97 & 260 & 25 & 180 & 16 & 260 & 27 & 130 & 360 \\
\hline 56 & 2.23 & 52 & 0.32 & 6.9 & 4.2 & 0.14 & 0.13 & 130 & 3100 & 15 & 75 & 210 & 35 & 240 & 18 & 210 & 22 & 125 & 350 \\
\hline 57 & 2.60 & 23 & 0.084 & 1.6 & 8.9 & 2.6 & 0.47 & 90 & 4100 & 34 & 10 & 590 & 15 & 130 & 4 & 190 & 22 & 110 & 280 \\
\hline 58 & 0.74 & 2.9 & 0.0054 & 0.090 & 1.9 & 0.56 & 0.12 & 45 & 760 & 8 & $<10$ & 90 & $<10$ & 34 & $<3$ & 42 & 13 & 17 & 70 \\
\hline 59 & 0.37 & 2.2 & 0.0036 & 0.090 & 1.9 & 0.58 & 0.11 & 40 & 600 & 7 & $<10$ & 82 & $<10$ & 41 & $<3$ & 39 & 12 & 18 & 70 \\
\hline 60 & 0.70 & 2.2 & 0.0030 & 0.074 & 1.5 & 0.41 & 0.092 & 42 & 650 & 8 & $<10$ & 80 & $<10$ & 30 & $<3$ & 26 & 13 & 14 & 40 \\
\hline 61 & 0.70 & 1.8 & 0.0033 & 0.079 & 1.5 & 0.43 & 0.092 & 45 & 650 & 9 & $<10$ & 70 & $<10$ & 32 & $<3$ & 26 & 13 & 13 & 60 \\
\hline 62 & 0.82 & 3.3 & 0.0078 & 0.12 & 1.6 & 0.50 & 0.11 & 90 & 730 & 10 & $<10$ & 105 & $<10$ & 51 & $<3$ & 27 & 14 & 16 & 50 \\
\hline 63 & 0.65 & 2.4 & 0.0072 & 0.095 & 1.5 & 0.31 & 0.10 & 35 & 810 & 7 & $<10$ & 75 & $<10$ & 33 & $<3$ & 57 & 7 & 16 & 40 \\
\hline 64 & 0.70 & 6.1 & 0.010 & 0.18 & 2.6 & 0.85 & 0.12 & 46 & 580 & 11 & $<10$ & 130 & $<10$ & 72 & $<3$ & 75 & 11 & 20 & 80 \\
\hline 65 & 0.46 & 4.1 & 0.011 & 0.14 & 2.2 & 0.96 & 0.14 & 48 & 810 & 8 & 28 & 160 & $<10$ & 80 & $<3$ & 85 & 12 & 20 & 80 \\
\hline 66 & 0.78 & 2.9 & 0.011 & 0.14 & 2.0 & 0.76 & 0.19 & 40 & 600 & 7 & $<10$ & 77 & $<10$ & 42 & $<3$ & 80 & 8 & 20 & 70 \\
\hline
\end{tabular}


TABLE 2 - Continued

\begin{tabular}{|c|c|c|c|c|c|c|c|c|c|c|c|c|c|c|c|c|c|c|c|}
\hline $\begin{array}{l}\text { Sample } \\
\text { No. }\end{array}$ & $\begin{array}{l}\mathrm{Na} \\
(\%)\end{array}$ & $\begin{array}{l}\mathrm{SiO}_{2} \\
(\%)\end{array}$ & $\begin{array}{l}\mathrm{Ti} \\
(\%)\end{array}$ & $\begin{array}{l}\mathrm{Al} \\
(\%)\end{array}$ & $\begin{array}{l}\mathrm{Fe} \\
(\%)\end{array}$ & $\begin{array}{l}\mathrm{Mn} \\
(\%)\end{array}$ & $\begin{array}{c}\mathrm{P}_{2} \mathrm{O}_{5} \\
(\%)\end{array}$ & $\underset{(\mathrm{ppm})}{\mathrm{B}}$ & $\underset{(\mathrm{ppm})}{\mathrm{Ba}}$ & $\begin{array}{c}\text { Co } \\
\text { (ppm) }\end{array}$ & $\begin{array}{c}\mathrm{Cr} \\
(\mathrm{ppm})\end{array}$ & $\begin{array}{c}\mathrm{Cu} \\
(\mathrm{ppm})\end{array}$ & $\begin{array}{c}\mathrm{La} \\
(\mathrm{ppm})\end{array}$ & $\begin{array}{c}\mathrm{Ni} \\
(\mathrm{ppm})\end{array}$ & $\begin{array}{c}\mathrm{Sc} \\
(\mathrm{ppm})\end{array}$ & $\begin{array}{c}\mathrm{V} \\
(\mathrm{ppm})\end{array}$ & $\begin{array}{c}\mathrm{Y} \\
(\mathrm{ppm})\end{array}$ & $\begin{array}{c}\mathrm{Zr} \\
(\mathrm{ppm})\end{array}$ & $\underset{(\mathrm{ppm})}{\mathrm{Zn}}$ \\
\hline 67 & 0.67 & 2.9 & 0.078 & 0.11 & 2.0 & 0.79 & 0.17 & 100 & 650 & 7 & $<10$ & 135 & $<10$ & 75 & $<3$ & 80 & 8 & 18 & 60 \\
\hline 68 & 0.63 & 2.2 & 0.011 & 0.11 & 3.0 & 0.91 & 0.22 & 45 & 610 & 11 & $<10$ & 160 & $<10$ & 68 & $<3$ & 110 & 13 & 20 & 60 \\
\hline 69 & 0.41 & 3.3 & 0.0090 & 0.10 & 1.6 & 0.70 & 0.18 & 48 & 810 & 7 & $<10$ & 89 & $<10$ & 53 & $<3$ & 70 & 9 & 22 & 50 \\
\hline 70 & 3.71 & 52 & 0.30 & 8.0 & 4.9 & 0.051 & 0.18 & 105 & 3400 & 24 & 86 & 210 & 32 & 130 & 17 & 160 & 45 & 160 & 180 \\
\hline 71 & 2.37 & 57 & 0.53 & 7.7 & 5.2 & 0.053 & 0.21 & 105 & 3700 & 22 & 78 & 290 & 30 & 68 & 17 & 210 & 45 & 190 & 160 \\
\hline 72 & 3.71 & 54 & 0.35 & 7.4 & 4.7 & 0.050 & 0.17 & 105 & 3400 & 36 & 77 & 280 & 52 & 120 & 16 & 180 & 40 & 160 & 160 \\
\hline 73 & 4.23 & 54 & 0.31 & 7.2 & 4.7 & 0.093 & 0.14 & 110 & 3400 & 110 & 90 & 1300 & 54 & 330 & 18 & 190 & 40 & 170 & 270 \\
\hline 74 & 2.40 & 52 & 0.34 & 7.5 & 5.2 & 0.071 & 0.21 & 110 & 4100 & 52 & 105 & 590 & 53 & 260 & 22 & 155 & 52 & 175 & 220 \\
\hline 75 & 3.71 & 55 & 0.35 & 6.8 & 4.8 & 0.10 & 0.24 & 110 & 4900 & 52 & 88 & 590 & 60 & 340 & 16 & 105 & 70 & 175 & 210 \\
\hline 76 & 4.30 & 48 & 0.29 & 6.4 & 4.8 & 0.37 & 0.39 & 90 & 4600 & 47 & 90 & 510 & 52 & 300 & 12 & 100 & 77 & 150 & 230 \\
\hline 77 & 4.45 & 52 & 0.31 & 5.9 & 6.0 & 0.25 & 0.35 & 110 & 1100 & 45 & 120 & 530 & 60 & 370 & 17 & 100 & 69 & 190 & 260 \\
\hline 78 & 3.71 & 45 & 0.32 & 6.1 & 6.4 & 0.19 & 0.39 & 100 & 8200 & 50 & 115 & 610 & 82 & 440 & 36 & 110 & 86 & 170 & 280 \\
\hline 79 & 3.71 & 51 & 0.30 & 6.1 & 6.1 & 0.71 & 0.36 & 105 & 5200 & 130 & 90 & 640 & 85 & 440 & 14 & 100 & 88 & 175 & 190 \\
\hline 80 & 3.93 & 51 & 0.28 & 6.4 & 6.6 & 2.2 & 0.99 & 110 & 7600 & 180 & 25 & 720 & 170 & 390 & 29 & 110 & 230 & 200 & 270 \\
\hline 81 & 3.00 & 50 & 0.33 & 6.6 & 7.7 & 3.1 & 1.38 & 110 & 4100 & 320 & 31 & 690 & 190 & 460 & 36 & 190 & 280 & 260 & 240 \\
\hline 82 & 2.23 & 36 & 0.30 & 4.3 & 13.7 & 4.2 & 2.0 & 140 & 1900 & 190 & 27 & 830 & 210 & 620 & 26 & 170 & 240 & 220 & 450 \\
\hline 83 & 0.60 & 1.5 & 0.0084 & 0.19 & 0.41 & 0.12 & 0.089 & 55 & 240 & $<5$ & $<10$ & 290 & $<10$ & 19 & $<3$ & 6 & 8 & 14 & 70 \\
\hline 84 & 2.08 & 24 & 0.17 & 3.7 & 7.8 & 1.9 & 1.06 & 92 & 760 & 140 & 27 & 510 & 85 & 270 & 11 & 200 & 170 & 180 & 230 \\
\hline 85 & 1.14 & 8.2 & 0.060 & 1.3 & 7.8 & 1.4 & 0.62 & 90 & 360 & 48 & 16 & 440 & ND & 160 & 4 & 390 & 80 & 95 & 240 \\
\hline 86 & 0.76 & 5.2 & 0.066 & 1.5 & 9.6 & 1.9 & 0.74 & 80 & 330 & 75 & 11 & 410 & 65 & 260 & 7 & 220 & 40 & 110 & 270 \\
\hline 87 & 0.73 & 1.9 & 0.0090 & 0.22 & 1.3 & 0.31 & 0.16 & 82 & 120 & 7.5 & $<10$ & 50 & $<10$ & 22 & $<3$ & 27 & 17 & 25 & 50 \\
\hline 88 & 0.56 & 1.8 & 0.0078 & 0.19 & 0.77 & 0.20 & 0.11 & 40 & 220 & 4 & $<10$ & 35 & $<10$ & 14 & $<3$ & 13 & 12 & 17 & 40 \\
\hline 89 & 0.32 & 2.0 & 0.0096 & 0.22 & 0.92 & 0.25 & 0.14 & 30 & 370 & 4 & $<10$ & 32 & $<10$ & 13 & $<3$ & 14 & 30 & 22 & 40 \\
\hline 90 & 0.70 & 3.9 & 0.037 & 0.40 & 4.6 & 2.6 & 0.29 & 45 & 550 & 13 & $<10$ & 320 & ND & 180 & $<3$ & 170 & 55 & 45 & 190 \\
\hline 91 & 0.50 & 2.6 & 0.022 & 0.26 & 5.4 & 2.8 & 0.29 & 60 & 340 & 22 & $<10$ & 260 & ND & 240 & $<3$ & 170 & 28 & 35 & 180 \\
\hline 92 & 0.95 & 2.7 & 0.051 & 0.28 & 4.7 & 2.3 & 0.27 & 60 & 310 & 13 & $<10$ & 240 & ND & 160 & $<3$ & 170 & 19 & 35 & 160 \\
\hline 93 & 0.82 & 3.5 & 0.057 & 0.30 & 4.4 & 2.0 & 0.29 & 110 & 330 & 17 & 10 & 260 & ND & 190 & 3 & 190 & 20 & 30 & 140 \\
\hline 94 & 1.00 & 6.5 & 0.096 & 0.51 & 3.7 & 1.4 & 0.26 & 60 & 300 & 17 & 10 & 180 & ND & 140 & 4 & 160 & 15 & 29 & 110 \\
\hline 95 & 1.30 & 15.5 & 0.22 & 1.01 & 5.0 & 1.6 & 0.18 & 80 & 420 & 28 & 15 & 180 & ND & 170 & 5 & 130 & 16 & 28 & 140 \\
\hline
\end{tabular}

Note: All values are given on an absolute basis in wt $\%$ or weight ppm for samples dried at $100^{\circ} \mathrm{C}$; that is, no correction for carbonate content has been made. NA $=$ not analyzed (for instance, due to los of samples). $\mathrm{ND}=$ not determined (for instance, due to severe interference from other spectral lines). Values given for double samples (for instance, $3 \& 4,6 \& 7,10 \& 11$, etc) represent averages of two analyses of two aliquots of the same sampling spot in the section. 
TABLE 3

Sample Distribution, Physical Properties, Geochronology, and Accumulation Rates for Sediments Sampled During DSDP Leg 34

\begin{tabular}{|c|c|c|c|c|c|c|c|}
\hline Group & $\begin{array}{c}\text { Core } \\
\text { Section }\end{array}$ & $\begin{array}{l}\text { DISUD } \\
(\mathrm{g} / \mathrm{CC})\end{array}$ & $\begin{array}{l}\text { Layer } \\
\text { Below } \\
\text { Mud line } \\
\text { (m) }\end{array}$ & $\begin{array}{l}\text { Thickness } \\
\text { (m) }\end{array}$ & $\begin{array}{c}\text { Age } \\
\text { (m.y.) }\end{array}$ & $\begin{array}{l}\text { Sedimenta- } \\
\text { tion Rate } \\
(\mathrm{mm} / 1000 \mathrm{yr})\end{array}$ & $\begin{array}{c}\text { Accumulation } \\
\text { Rate of Sedi- } \\
\text { ment }\left(\mathrm{mg} / \mathrm{cm}^{2}\right. \\
1000 \mathrm{yr})\end{array}$ \\
\hline \multicolumn{8}{|c|}{ Hole 319} \\
\hline A (1) & $1-1$ & 0.33 & $0-2$ & 2 & $0-2$ & 1.0 & 33 \\
\hline B (13) & $1-2$ to $3-3$ & 0.41 & $2-23$ & 21 & $2-12$ & 2.0 & 86 \\
\hline C (13) & $3-3$ to 10 & 1.01 & $23-95$ & 72 & $12-14$ & 36 & 3600 \\
\hline $\mathrm{D}(21)$ & 11 and 12 & 1.10 & $95-111.5$ & 16.5 & $14-15$ & 16.5 & 1800 \\
\hline \multicolumn{8}{|c|}{ Hole 320} \\
\hline E (8) & 1 & 0.41 & $0-16$ & 16 & $0-2$ & 8 & 330 \\
\hline$F(5)$ & 2 and 3 & 0.97 & $>(74-112)$ & $>38$ & $15-20$ & $>7.6$ & $>740$ \\
\hline G (8) & $1 \mathrm{~B}$ and $2 \mathrm{~B}$ & 1.11 & $>(136-155)$ & $>19$ & $22-30$ & $>2.4$ & $>270$ \\
\hline \multicolumn{8}{|c|}{ Site 321} \\
\hline H (5) & 1 to 4 & 0.36 & $0-30$ & 30 & $0-2$ & 15 & 540 \\
\hline I (1) & 5 & 0.76 & $30-39.5$ & 9.5 & $2-2.5$ & 2.7 & 210 \\
\hline $\mathrm{J}(4)$ & 6 & 0.71 & $39.5-49$ & 9.5 & $5.5-12$ & 1.5 & 110 \\
\hline $\mathrm{K}(7)$ & 7 to 9 & 0.86 & $49-77.5$ & 28.5 & $22-35$ & 2.2 & 190 \\
\hline $\mathbf{L}(1)$ & 10 & 1.20 & $77.5-87$ & 9.5 & $35-36.5$ & 6.3 & 760 \\
\hline M (2) & 11,12 & 1.10 & $87-115.5$ & 28.5 & $36.5-38$ & 19.0 & 2090 \\
\hline N (6) & 13 & 1.02 & $115.5-125$ & 9.5 & $38-41$ & 3.2 & 330 \\
\hline
\end{tabular}

Note: Data in this table are based on chronological information (primarily foraminifera zones), bulk densities, porosities, and depth distribution of the samples that have been presented elsewhere in this volume and in Table 1. Furthermore, absolute-sedimentation rates were obtained using the relationships between relative and absolute ages (Berggren, 1969; and later revisions). Number in parentheses behind each group label (A, B, etc) represents number of analyses used for the averaging in Tables 4 to 6 . DISUD stands for dry in situ uncompressed density, in other words, the weight of the dry matter within a cc at the sampling depth. DISUD is easily calculated from the bulk densities and porosities presented elsewhere in this volume. Note that the incomplete coring of Hole 320 gives rise to very uncertain data for groups $\mathrm{F}$ and $\mathrm{G}$. Other uncertainties, for instance, whether groups L and $\mathrm{M}$ should be presented as a single group or separately, are caused by ambiguities in chronological descriptions. However, a combined group $(\mathrm{L}+\mathrm{M})$ would have an accumulation rate of about $1700 \mathrm{mg} / \mathrm{cm}^{2} 1000 \mathrm{yr}$; the overall accumulation rate patterns presented in this paper will therefore not change if $\mathrm{L}$ and $\mathrm{M}$ were combined. Group $\mathrm{M}$ may be particularly overestimated due to these uncertainties, but a study of Table 6 reveals that even if the accumulation rate data for $M$ are reduced with $50 \%$, this does not alter the overall conclusions in this paper. Likewise, data for group N might be somewhat underestimated; if corresponding "correction" is included in Table 6 the conclusions in this paper are even more reinforced. It is therefore obvious that minor uncertainties in the accumulation rate data do not significantly affect the results in this paper, but they are serious enough to prevent a far-reaching in detail discussion of what has happened with the sediments, as is clearly demonstrated in the section of the paper that discusses paleophysiographic problems.

that with increased spreading rates more intense heat flow also takes place through the ocean floor. The higher heat flow should result in stronger hydrothermal leaching than is possible in areas of low heat flow. However, studies of hot springs in basaltic areas suggest that there is no significant differentiation between $\mathrm{Fe}$ and $\mathrm{Al}$, that is, the formed solutions contain equal quantities of $\mathrm{Al}$ and $\mathrm{Fe}$. The same conclusion is obtained from experimental leaching experiments involving basaltic matter and weak to strong $\mathrm{NaCl}$ brines; also, there is no significant segregation of the elements $\mathrm{Fe}, \mathrm{Mn}, \mathrm{Al}$, and $\mathrm{Ti}$ during the magmatic or effusive phases that emplace basalt in shallow regions of the oceanic crust. Furthermore, it does not appear likely that spilitization of lavas would give rise to $\mathrm{Fe}-\mathrm{Mn}$-rich, $\mathrm{Al}-\mathrm{Si}$-Ti-poor solutions. Carbonate-rich emanations, on the other hand, tend to be enriched in $\mathrm{Fe}, \mathrm{Mn}, \mathrm{P}, \mathrm{Ba}$, and $\mathrm{U}$ and depleted in $\mathrm{Al}$, $\mathrm{Ti}, \mathrm{Si}$, and $\mathrm{Th}$. It is, therefore, possible that carbonate- rich emanations from the mantle may play a major role in the separation of these elements. Green (1972) has shown that much $\mathrm{CO}_{2}$ may indeed emanate from the mantle. The problems discussed above in this section have been reviewed at length by Boström (1973).

The discussion here has referred to the major element distribution in the sediments. Data in Boström et al., 1973a, Boström, in press) give additional support to the conclusion that the influx of terrigenous matter and some addition of an EPR constituent (see Figures 1 and 2) control the main element composition of the sediments, whereas sources of basaltic detritus are less significant.

\section{Origin of Trace Constituents in the Basal Sediments}

The sources for the trace elements in the East Pacific sediments are less easily determined. For this discussion we will use data in Tables 7 and 8 and graphical displays 
TABLE 4

Average Chemical Compositions of Sediments Recovered During DSDP Leg 34

\begin{tabular}{|c|c|c|c|c|c|c|c|c|c|c|c|c|c|c|c|c|c|c|}
\hline Group & $\begin{array}{c}\mathrm{SiO}_{2} \\
(\%)\end{array}$ & $\begin{array}{c}\mathrm{Ti} \\
(\%)\end{array}$ & $\begin{array}{r}\mathrm{Al} \\
(\%)\end{array}$ & $\begin{array}{c}\mathrm{Fe} \\
(\%)\end{array}$ & $\begin{array}{l}\mathrm{Mn} \\
(\%)\end{array}$ & $\begin{array}{c}\mathrm{P}_{2} \mathrm{O}_{5} \\
(\%)\end{array}$ & $\begin{array}{c}\text { B } \\
(\mathrm{ppm})\end{array}$ & $\begin{array}{c}\mathbf{B a} \\
(\mathrm{ppm})\end{array}$ & $\begin{array}{c}\text { Co } \\
\text { (ppm) }\end{array}$ & $\begin{array}{c}\mathrm{Cr} \\
(\mathrm{ppm})\end{array}$ & $\begin{array}{c}\mathrm{Cu} \\
(\mathrm{ppm})\end{array}$ & $\begin{array}{c}\mathrm{La} \\
(\mathrm{ppm})\end{array}$ & $\begin{array}{c}\mathrm{Ni} \\
(\mathrm{ppm})\end{array}$ & $\begin{array}{c}\mathrm{Sc} \\
(\mathrm{ppm})\end{array}$ & $\begin{array}{c}\mathrm{V} \\
(\mathrm{ppm})\end{array}$ & $\begin{array}{c}\mathrm{Y} \\
(\mathrm{ppm})\end{array}$ & $\begin{array}{c}\mathrm{Zr} \\
\text { (ppm) }\end{array}$ & $\begin{array}{c}\mathrm{Zn} \\
(\mathrm{ppm})\end{array}$ \\
\hline \multicolumn{19}{|c|}{ Hole 319} \\
\hline A & 29 & 0.16 & 2.5 & 13.3 & 4.0 & 2.1 & 150 & 9100 & 220 & 13 & 850 & 280 & 540 & 17 & 190 & 1200 & 720 & 800 \\
\hline B & 18 & 0.10 & 1.1 & 10.1 & 2.2 & 0.96 & 106 & 6400 & 93 & $\sim 6$ & 640 & 110 & 280 & $\sim 7$ & 175 & 270 & 160 & 250 \\
\hline C & 1.7 & 0.0088 & 0.14 & 2.44 & 0.95 & 0.24 & 50 & 860 & 16 & $<5$ & 180 & $\sim 18$ & 69 & $\sim 3$ & 94 & 28 & 26 & 110 \\
\hline D & 2.0 & 0.0097 & 0.14 & 2.18 & 0.64 & 0.27 & 46 & 820 & 15 & $<5$ & 116 & $<10$ & 54 & $<2$ & 60 & 19 & 20 & 73 \\
\hline \multicolumn{19}{|c|}{ Hole 320} \\
\hline $\mathbf{E}$ & 51 & 0.35 & 7.9 & 4.35 & 0.17 & 0.15 & 114 & 3300 & 17 & 78 & 250 & 28 & 220 & 16 & 215 & 23 & 120 & 320 \\
\hline F & 6.4 & 0.020 & 0.38 & 3.12 & 0.91 & 0.18 & 52 & 1350 & 13 & $<10$ & 180 & $<10$ & 53 & $<3$ & 65 & 15 & 34 & 104 \\
\hline G & 3.4 & 0.0093 & 0.12 & 2.06 & 0.72 & 0.15 & 57 & 700 & 8.5 & $<10$ & 116 & $<10$ & 59 & $<3$ & 73 & 10 & 19 & 61 \\
\hline \multicolumn{19}{|c|}{ Site 321} \\
\hline H & 54 & 0.37 & 7.6 & 4.93 & 0.064 & 0.18 & 107 & 3600 & 49 & 87 & 535 & 44 & 180 & 18 & 180 & 44 & 170 & 200 \\
\hline I & 55 & 0.35 & 6.8 & 4.80 & 0.10 & 0.24 & 110 & 4900 & 52 & 88 & 590 & 60 & 340 & 16 & 105 & 70 & 175 & 210 \\
\hline $\mathbf{J}$ & 49 & 0.31 & 6.1 & 5.81 & 0.38 & 0.37 & 100 & 4800 & 68 & 105 & 575 & 70 & 390 & 18 & 105 & 80 & 172 & 240 \\
\hline $\mathrm{K}$ & 25 & 0.17 & 3.4 & 7.65 & 2.10 & 0.99 & 97 & 2200 & 137 & 20 & 555 & 120 & 310 & 16 & 185 & 150 & 155 & 255 \\
\hline L & 1.9 & 0.0090 & 0.22 & 1.30 & 0.31 & 0.16 & 82 & 120 & 7.5 & $<10$ & 50 & $<10$ & 22 & $<3$ & 27 & 17 & 25 & 50 \\
\hline M & 1.9 & 0.0087 & 0.21 & 0.85 & 0.23 & 0.13 & 35 & 295 & 4 & $<10$ & 34 & $<10$ & 14 & $<3$ & 14 & 21 & 20 & 40 \\
\hline $\mathrm{N}$ & 5.8 & 0.081 & 0.46 & 4.63 & 2.1 & 0.26 & 69 & 375 & 18 & $\sim 8.3$ & 240 & ND & 180 & $\sim 2.8$ & 165 & 26 & 34 & 153 \\
\hline
\end{tabular}

Note: All values are on an absolute basis; that is, no correction for $\mathrm{CaCO}_{3}$-content has been made. Much of the variations are due to the diluting effect of the CaCO 3 content. For definitions of groups A to N, see Table 3 . 
TABLE 5

Compositions of Carbonate- and Opaline Silica-Free Fractions of Sediments Recovered During DSDP Leg 34

\begin{tabular}{|c|c|c|c|c|c|c|c|c|c|c|c|c|c|c|c|c|c|}
\hline Group & $\begin{array}{c}\mathrm{Ti} \\
(\%)\end{array}$ & $\begin{array}{c}\mathrm{Al} \\
(\%)\end{array}$ & $\begin{array}{l}\mathrm{Fe} \\
(\%)\end{array}$ & $\begin{array}{l}\mathrm{Mn} \\
(\%)\end{array}$ & $\begin{array}{c}\mathrm{P}_{2} \mathrm{O}_{5} \\
(\%)\end{array}$ & $\begin{array}{c}\mathrm{B} \\
(\mathrm{ppm})\end{array}$ & $\begin{array}{c}\mathrm{Ba} \\
(\mathrm{ppm})\end{array}$ & $\begin{array}{c}\mathrm{Co} \\
(\mathrm{ppm})\end{array}$ & $\begin{array}{c}\mathrm{Cr} \\
(\mathrm{ppm})\end{array}$ & $\underset{(\mathrm{ppm})}{\mathrm{Cu}}$ & $\begin{array}{c}\mathrm{La} \\
(\mathrm{ppm})\end{array}$ & $\underset{(\mathrm{ppm})}{\mathrm{Ni}}$ & $\underset{(\mathrm{ppm})}{\mathrm{Sc}}$ & $\begin{array}{c}\mathrm{V} \\
(\mathrm{ppm})\end{array}$ & $\begin{array}{c}\mathrm{Y} \\
(\mathrm{ppm})\end{array}$ & $\begin{array}{c}\mathrm{Zr} \\
(\mathrm{ppm})\end{array}$ & $\begin{array}{c}\mathrm{Zn} \\
(\mathrm{ppm})\end{array}$ \\
\hline \multicolumn{18}{|l|}{ Hole 319} \\
\hline A & 0.21 & 3.3 & 17.5 & 5.3 & 2.8 & 195 & 12,000 & 290 & 17 & 1100 & 370 & 710 & 22 & 250 & 1600 & 945 & 1050 \\
\hline B & 0.23 & 2.4 & 22.0 & 4.8 & 2.1 & 230 & 14,000 & 200 & $\sim 13$ & 1400 & 240 & 610 & $\sim 15$ & 380 & 585 & 350 & 545 \\
\hline C & 0.083 & 1.3 & 23.0 & 9.0 & 2.3 & 470 & 8,100 & 150 & - & 1700 & $\sim 170$ & 650 & $\sim 28$ & 885 & 265 & 245 & 1040 \\
\hline $\mathrm{D}$ & 0.10 & 1.5 & 23.3 & 6.8 & 2.9 & 490 & 8,800 & 160 & - & 1240 & - & 575 & - & 640 & 205 & 215 & 780 \\
\hline \multicolumn{18}{|l|}{ Hole 320} \\
\hline $\mathrm{E}$ & 0.38 & 8.6 & 4.71 & 0.18 & 0.16 & 125 & 3,600 & 18 & 84 & 270 & 30 & 240 & 17 & 235 & 27 & 130 & 345 \\
\hline $\mathrm{F}$ & 0.14 & 2.6 & 21.2 & 6.2 & 1.2 & 355 & 9,200 & 88 & - & 1200 & - & 360 & - & 440 & 100 & 230 & 705 \\
\hline G & 0.11 & 1.4 & 23.6 & 8.3 & 1.7 & 655 & 8,000 & 97 & - & 1300 & - & 675 & - & 835 & 115 & 220 & 700 \\
\hline \multicolumn{18}{|l|}{ Hole 321} \\
\hline $\mathrm{H}$ & 0.41 & 8.4 & 5.43 & 0.071 & 0.20 & 120 & 4,000 & 54 & 96 & 590 & 48 & 200 & 20 & 200 & 48 & 185 & 220 \\
\hline I & 0.42 & 8.2 & 5.79 & 0.12 & 0.29 & 135 & 5,900 & 63 & 105 & 710 & 72 & 410 & 19 & 125 & 84 & 210 & 255 \\
\hline $\mathrm{J}$ & 0.35 & 6.8 & 6.51 & 0.43 & 0.41 & 112 & 5,400 & 76 & 120 & 645 & 78 & 435 & 20 & 120 & 90 & 195 & 270 \\
\hline K & 0.28 & 5.5 & 12.4 & 3.4 & 1.6 & 155 & 3,600 & 220 & 32 & 895 & 195 & 500 & 26 & 300 & 240 & 250 & 410 \\
\hline L & 0.14 & 3.3 & 19.5 & 4.6 & 2.4 & 1200 & 1,800 & 115 & - & 750 & - & 330 & - & 405 & 255 & 375 & 750 \\
\hline M & 0.17 & 4.0 & 16.3 & 4.4 & 2.5 & 670 & 5,700 & 77 & - & 650 & - & 270 & - & 270 & 400 & 385 & 765 \\
\hline $\mathrm{N}$ & 0.36 & 2.1 & 20.7 & 9.4 & 1.2 & 310 & 1,700 & 80 & $\sim 37$ & 1100 & ND & 805 & 13 & 740 & 115 & 150 & 685 \\
\hline GS 7202-35 & 0.030 & 0.29 & 25.2 & 9.5 & 3.7 & 725 & 3,700 & 86 & - & 1310 & 100 & 600 & 2.9 & 840 & 115 & 130 & 570 \\
\hline
\end{tabular}

Note: For definition of groups A to N, see Table 3. The lowest line shows data for core GS 7202-35 (see also caption to Table 6 and Boström et al., 1974a). This table shows the normalized values from Table 4, obtained by the multiplication of the Ti, Al, Fe ..., Zn values for each group $i$ with a factor $F_{i}$, defined as follows:

$$
F_{i}=\frac{40}{\left(4 \mathrm{Al}_{i}+\mathrm{Ti}_{i}+\mathrm{Fe}_{i}+\mathrm{Mn}_{i}+\mathrm{P}_{2} \mathrm{O}_{5} i+\mathrm{Ba}_{i}\right)}
$$

(all input data given in wt \%). This expression is justified by the following facts: (a) In well-analyzed sediments with no opaline silica the sum of the oxides, hydroxides, and other compounds of $\mathrm{Si}, \mathrm{Al}, \mathrm{Ti}, \mathrm{Fe}, \mathrm{Mn}, \mathrm{P}$, and $\mathrm{Ba}$ constitute about $85 \%$ of the carbonate-free fraction or about half that amount ( $\sim 40 \%)$ that concerns the sum of the elements $\mathrm{Si}$, $\mathrm{Al}$, Ti, etc. (b) In sediments with little or no opaline silica the ratio $\mathrm{SiO}_{2} / \mathrm{Al}_{2} \mathrm{O}_{3}$ usually ranges between 3 and 4 , the ratio found in average continental crustal matter (see Table 8 ); that is, the inorganic $\mathrm{Si}_{i} \approx 3 \mathrm{Al}_{i}$ in the sediments. In the expression for $\mathrm{F}_{i}$ above, $3 \mathrm{Al}_{i}$ thus stand for the inorganic $\mathrm{Si}$ fraction and $4 \mathrm{Al}_{i}$ thus for the combined $\mathrm{Si}_{i}$ (inorg.) and $\mathrm{Al}_{i}$ contributions. The best test of a method is to check whether it works or not. In carefully analyzed deep-sea sediments with much terrigenous matter Al usually ranges between $8 \%-9 \%$, Fe usually between $4 \%-5 \%$, and B between $110-135 \mathrm{ppm}$ (Landergren, 1964). These values compare well with respective values for groups E, H, and I, which show heavy admix ture of terrigenous matter. A more detailed discussion of the need for sediment petrographic normalization procedures is presented in Boström et al., 1972b. However, the method tends to punish sediment data from active ridges; thus the average values for core GS7202-35 on the lowest line would be about $10 \%$ higher if a recalculation is made using obtained CaCO 3 , $\mathrm{NaC} 1, \mathrm{KC} 1, \mathrm{MgCl}_{2}$, and $\mathrm{SrCO}_{3}$ concentrations. 
TABLE 6

Accumulation Rates for Constituents in Sediments Recovered During DSDP Leg 34

\begin{tabular}{|c|c|c|c|c|c|c|c|c|c|c|c|c|c|c|c|c|c|c|c|c|}
\hline Group & $\mathrm{SiO}_{2}$ & $\mathrm{SiO}_{2} *$ & $\mathrm{Ti}$ & $\mathrm{Al}$ & $\mathrm{Fe}$ & $\mathrm{Fe}^{*}$ & $\mathrm{Mn}$ & $\mathrm{P}_{2} \mathrm{O}_{5}$ & $\mathrm{Ba}$ & B & $\mathrm{Co}$ & $\mathrm{Cr}$ & $\mathrm{Cu}$ & $\mathrm{La}$ & $\mathrm{Ni}$ & $\mathrm{Sc}$ & V & $\mathrm{Y}$ & $\mathrm{Zr}$ & $\mathrm{Zn}$ \\
\hline Hole 319 & \multicolumn{9}{|c|}{ (in $\mathrm{mg} / \mathrm{cm}^{2} 1000 \mathrm{yr}$ ) } & \multicolumn{11}{|c|}{ (in $\mathrm{mg} / \mathrm{cm}^{2} 1,000,000 \mathrm{yr}$ ) } \\
\hline $\mathbf{A}$ & 9.6 & 3.5 & 0.053 & 0.83 & 4.4 & 3.9 & 1.3 & 0.69 & 0.30 & 5 & 7 & 0.4 & 28 & 9 & 18 & 0.6 & 6 & 40 & 24 & 26 \\
\hline B & 16 & 8.5 & 0.086 & 0.95 & 8.7 & 8.1 & 1.9 & 0.83 & 0.55 & 9 & 8 & 0.5 & 55 & 10 & 24 & 0.6 & 15 & 23 & 14 & 22 \\
\hline C & 61 & 24 & 0.32 & 5.0 & 88.0 & 85.0 & 34 & 8.6 & 3.1 & 180 & 58 & $<18$ & 650 & 65 & 250 & 11 & 340 & 100 & 94 & 400 \\
\hline $\mathrm{D}$ & 36 & 17 & 0.17 & 2.5 & 39.0 & 38.0 & 12 & 4.9 & 1.5 & 83 & 27 & $<9$ & 210 & $<18$ & 97 & $<3.6$ & 110 & 34 & 36 & 130 \\
\hline \multicolumn{21}{|l|}{ Hole 320} \\
\hline E & 168 & -25 & 1.2 & 26 & 14.0 & -1.6 & 0.56 & 0.50 & 1.1 & 38 & 6 & 26 & 83 & 9 & 73 & 5 & 71 & 8 & 40 & 110 \\
\hline G & 9.2 & 6.8 & 0.025 & 0.32 & 5.6 & 5.4 & 1.9 & 0.41 & 0.19 & 15 & 2 & $?$ & 31 & $?$ & 16 & $?$ & 20 & 3 & 5 & 17 \\
\hline \multicolumn{21}{|l|}{ Site 321} \\
\hline $\mathbf{H}$ & 290 & -13 & 2.0 & 41 & 27.0 & 2.4 & 0.35 & 0.97 & 1.9 & 58 & 27 & 47 & 290 & 24 & 97 & 8 & 97 & 24 & 92 & 110 \\
\hline I & 116 & 12 & 0.74 & 14 & 10.0 & 1.6 & 0.21 & 0.50 & 1.0 & 23 & 11 & 19 & 120 & 13 & 71 & 3 & 22 & 15 & 38 & 44 \\
\hline $\mathrm{J}$ & 54 & 4.4 & 0.34 & 6.7 & 6.4 & 2.4 & 0.42 & 0.41 & 0.53 & 11 & 8 & 12 & 63 & 8 & 43 & 2 & 12 & 9 & 19 & 26 \\
\hline K & 48 & 0 & 0.32 & 6.5 & 15.0 & 11.0 & 4.0 & 1.9 & 0.42 & 18 & 27 & 4 & 110 & 23 & 59 & 3 & 35 & 29 & 30 & 49 \\
\hline L & 14 & 1.4 & 0.014 & 1.7 & 9.9 & 8.9 & 2.4 & 1.2 & 0.09 & 62 & 6 & $<8$ & 38 & $<8$ & 17 & $<2$ & 21 & 13 & 19 & 38 \\
\hline M & 40 & 7.4 & 0.18 & 4.4 & 18.0 & 15.0 & 4.8 & 2.7 & 0.62 & 73 & 8 & $<21$ & 71 & 21 & 29 & 6 & 29 & 44 & 42 & 84 \\
\hline $\mathrm{N}$ & 19 & 7.9 & 0.27 & 1.5 & 15.0 & 14.0 & 6.9 & 0.88 & 0.13 & 23 & 6 & 3 & 80 & - & 60 & 0.9 & 55 & 9 & 11 & 51 \\
\hline
\end{tabular}

Note: Values for groups $\mathrm{F}$ and $\mathrm{G}$ may be too low. $\mathrm{SiO}_{2}$ * represents opaline silica, which is the quantity of $\mathrm{SiO}_{2}$ that exceeds the value to be expected from the ratio $\mathrm{SiO}_{2} / \mathrm{Al}$ in continental matter (Table 8). Fe* represents authigenic iron, which is the quantity of $\mathrm{Fe}$ that exceeds the value to be expected from the ratio $\mathrm{Fe} / \mathrm{Al}$ in continental matter (Table 8). Negative values for $\mathrm{Fe}^{*}$ and $\mathrm{SiO}_{2} *$ implies losses due to weathering; characteristically these deficits occur in samples that are rich in terrigenous matter deposited close to the continents (groups $\mathrm{E}$ and $\mathrm{H}$ ). In Hole 319 and Site 321 the deposits with the highest accumulation rates for Fe* invariably occur in the basal part of the sediment column. The lowest line shows the accumulation rates in core GS 7202-35, which was obtained very close to the spreading center on the present East Pacific Rise Position: $14^{\circ} 47.9^{\prime} \mathrm{S}, 113^{\circ} 30.1^{\prime} \mathrm{W}$, depth $3044 \mathrm{~m}$ (corrected), total core length $8.5 \mathrm{~m}$; for details, see Boström et al., 1974. 
TABLE 7

Average Accumulation Rates and Compositions of Sediments Rich

in "Volcanic" Matter (I) and in Terrigenous Matter (II), Based on Material from DSDP Leg 34

\begin{tabular}{|c|c|c|c|c|}
\hline \multicolumn{3}{|c|}{$\mathrm{I}^{\mathrm{a}}$} & \multicolumn{2}{|c|}{$\mathrm{II}^{\mathrm{b}}$} \\
\hline & $\begin{array}{c}\text { Average } \\
\text { Accumulation } \\
\text { Rate }\end{array}$ & $\begin{array}{c}\text { Average } \\
\text { Composition } \\
\left(\mathrm{CaCO}_{3} \&\right. \\
\mathrm{NaCl} \text { Free })\end{array}$ & $\begin{array}{c}\text { Average } \\
\text { Accumulation } \\
\text { Rate }\end{array}$ & $\begin{array}{c}\text { Average } \\
\text { Composition } \\
\left(\mathrm{CaCO}_{3} \&\right. \\
\mathrm{NaCl} \text { Free })\end{array}$ \\
\hline $\mathrm{SiO}_{2}$ & 30.5 & 23 & 157 & 57 \\
\hline $\mathrm{SiO}_{2} *$ & 12.1 & 8.5 & 19 & 6.8 \\
\hline $\mathrm{Ti}$ & 0.18 & 0.14 & 1.1 & 0.39 \\
\hline $\mathrm{Al}$ & 2.9 & 2.3 & 21.9 & 7.9 \\
\hline $\mathrm{Fe}$ & 24.8 & 19.1 & 14.4 & 5.2 \\
\hline $\mathrm{Fe}^{*}$ & 23.1 & 17.8 & 1.2 & 0.43 \\
\hline $\mathrm{Mn}$ & 8.4 & 6.5 & 0.39 & 0.14 \\
\hline $\mathrm{P}_{2} \mathrm{O}_{5}$ & 2.7 & 2.1 & 0.60 & 0.22 \\
\hline $\mathrm{Ba}$ & 0.84 & 0.65 & 1.1 & 0.41 \\
\hline B & 57 & 440 & 32 & 115 \\
\hline $\mathrm{Co}$ & 18 & 140 & 13 & 47 \\
\hline $\mathrm{Cu}$ & 155 & 1200 & 139 & 500 \\
\hline $\mathrm{Ni}$ & 112 & 860 & 71 & 260 \\
\hline $\mathrm{Sc}$ & $\approx 3.1$ & $\approx 24$ & 4.5 & 16 \\
\hline V & 76 & 590 & 50 & 180 \\
\hline $\mathrm{Y}$ & 37 & 285 & 14 & 50 \\
\hline $\mathrm{Zr}$ & 35 & 270 & 47 & 169 \\
\hline $\mathrm{Zn}$ & 100 & 770 & 73 & 260 \\
\hline
\end{tabular}

Note: Data for the major constituents $\mathrm{SiO}_{2}$ to $\mathrm{Ba}$ are given in $\mathrm{mg} / \mathrm{cm}^{2} 1000$ yr and wt \%, respectively, and for the trace constituents $\mathrm{B}$ to $\mathrm{Zr}$ in $\mathrm{mg} / \mathrm{cm}^{2} 1,000,000 \mathrm{yr}$ and wt ppm, respectively. The average compositions for the sediments are obtained from the average accumulation rates, using the assumption that the sum of the oxides, hydroxides, sulfates, and $\mathrm{Ca}$ compounds of the major constituents $\mathrm{SiO}_{2}, \mathrm{Ti}, \mathrm{Al}, \mathrm{Fe}$, $\mathrm{Mn}, \mathrm{P}_{2} \mathrm{O}_{5}$, and $\mathrm{Ba}$ constitute $85 \%$ of the $\mathrm{CaCO}_{3}$ - and $\mathrm{NaCl}$-free fraction of the sediments.

${ }^{a}$ The accumulation rates and composition of the basal sediments, rich in "volcanic" matter, are based on data in Table 6 for groups A, B, C, D, K, $\mathrm{L}, \mathrm{M}$, and $\mathrm{N}$, that is, for sediments that were emplaced within $15 \mathrm{~m} . \mathrm{y}$. of the formation of the oceanic crust.

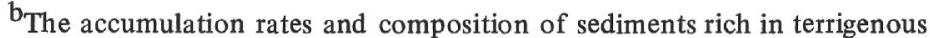
matter are based on data in Table 6 for groups E, H, I, and J, that is, for sediments that were emplaced more than $15 \mathrm{~m} . \mathrm{y}$. after the formation of the oceanic crust.

in Figure 4. To some extent major constituents have to be discussed also in this section.

The basal sediments (group I of Table 7) are too rich in $\mathrm{Fe}, \mathrm{Mn}, \mathrm{Ba}, \mathrm{Cu}, \mathrm{Ni}, \mathrm{V}$, and $\mathrm{Zn}$ to be formed mainly from terrigenous matter (TM); see Figure 4a. Biological matter (BM) alone cannot explain their origin either, since $\mathrm{BM}$ has a distinctly different $\mathrm{Al} / \mathrm{Ti}$ ratio and is too poor in $\mathrm{Fe}, \mathrm{Mn}, \mathrm{V}$, and $\mathrm{Zr}$ (see Figure $4 \mathrm{~b}$ ). On the other hand, a sediment model consisting of $88 \%$ EPR matter (EPRD) and $12 \%$ TM gives a very good reproduction of the basal sediments (see Figure 4c). These proportions are well defined; already a shift to the proportions 3:1 for EPRD:TM gives a model sediment with distinctly poorer fit. Some addition (25\%) of BM does not alter the situation much, but an admixture of 50\% BM does give a poor fit (Figure $4 d$ ).
These findings, however, do not clarify the origin of the EPR constituents, which obviously are of importance when we discuss both the major and the trace element geochemistry of East Pacific sediments. Neither $\mathrm{BM}$ nor TM are sufficient sources of $\mathrm{Mn}, \mathrm{Fe}, \mathrm{Ba}, \mathrm{Ni}, \mathrm{B}$, and $\mathrm{Cu}$. Accumulation rate data for $\mathrm{Al}$ and $\mathrm{Ti}$, both for these sediments and for the Pacific in general (Table 6, this paper, and Boström, in press) demonstrate that TM must be a totally insufficient source for most trace constituents, since TM is poor in trace constituents. BM, on the other hand, is fairly rich in trace elements relative to $\mathrm{Al}$ and $\mathrm{Ti}$. One can therefore suspect that a mixture of $\mathrm{BM}$ and an unknown additional source (volcanic?) will give rise to EPRD as shown in Table 8. Figure 4e shows a possible solution to this problem. A consequence of this solution is that (compare Figures $4 c$ and $4 \mathrm{e}$ ) the 
TABLE 8

Compositions of Constituents in Model Sediments (in wt \% on an Absolute Basis)

\begin{tabular}{lcccl}
\hline & $\mathrm{BM}^{\mathrm{a}}$ & $\mathrm{TM}^{\mathrm{b}}$ & $\mathrm{VM}^{\mathrm{c}}$ & $\mathrm{EPRD}^{\mathrm{d}}$ \\
\hline $\mathrm{SiO}_{2}$ & 17.4 & 53.3 & - & 3.69 \\
$\mathrm{Al}$ & 0.28 & 8.1 & - & 0.083 \\
$\mathrm{Ti}$ & 0.037 & 0.48 & - & 0.0085 \\
$\mathrm{Fe}$ & 0.48 & 4.9 & 27 & 7.30 \\
$\mathrm{Mn}$ & 0.015 & 0.088 & 9 & 2.76 \\
$\mathrm{Ba}$ & 0.048 & 0.054 & 2.6 & 0.24 \\
$\mathrm{~B}$ & 0.039 & 0.0078 & - & 0.021 \\
$\mathrm{Cu}$ & 0.028 & 0.0057 & - & 0.038 \\
$\mathrm{Ni}$ & 0.0093 & 0.0090 & - & 0.017 \\
$\mathrm{~V}$ & 0.0020 & 0.013 & 0.050 & 0.025 \\
$\mathrm{Zn}$ & 0.089 & 0.0078 & - & 0.017 \\
$\mathrm{Zr}$ & 0.0025 & 0.019 & - & 0.0037 \\
$\mathrm{CaCO}$ & 32.2 & 7.3 & - & 67 \\
\hline
\end{tabular}

${ }^{a}$ BM: Composition of average dry marine biological matter, primarily plankton, based on a large number of analyses from the literature and laboratory work at RSMAS; for details, see Boström et al., 1974b and Moore et al., in preparation. The standard errors for most minor and trace constituents in biological matter is very small once the analy ses have been normalized; for a detailed account, see Boström et al., 1974b.

$\mathrm{b}_{\mathrm{TM}}$ : Composition of terrigenous matter, derived from the assumption that weathered continental matter consists to $75 \%$ of average shale and to $25 \%$ of average continental crust. These proportions are obtained from the observation that the surface of the continents are to $75 \%$ covered by sedimentary deposits. Crust and shale abundances are from Krauskopf (1967).

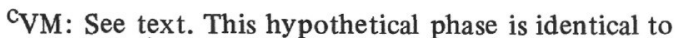
the one used in Boström, in press. The Ba-value is probably too high, but is difficult to estimate.

dEPRD: Composition of East Pacific Rise deposits, according to analyses of core GS 7202-35; similar values are reported for the Risepac cores 68 and 69 . (Boström et al., 1974a; Boström and Peterson, 1969.)

basal sediments are formed by $44 \%$ BM, $44 \%$ "volcanic" matter (VM), and 12\% TM. By using the same model inputs and calculations, one can find that the group II sediments (see Table 7) are produced by a mixture of about $74 \% \mathrm{BM}, 24 \% \mathrm{TM}$, and $2 \% \mathrm{VM}$.

The origin of this "volcanic" phase is not clear. It appears likely, in view of their high accumulations rates, that $\mathrm{Fe}$ and $\mathrm{Mn}$ must be delivered in association with the plate generating volcanism at spreading centers; and $\mathrm{Ba}, \mathrm{Cu}$, and $\mathrm{Ni}$, for instance, may be derived by absorption from seawater. It should be recalled, however, that elements which for quantitative reasons easily can be modeled as adsorbates, may nevertheless have a different source; this seems to be the case with $U$ (Rydell et al., 1974). Furthermore, some "adsorptionexplanations" may require very high removal efficiencies. Adsorption processes may indeed have been invoked too loosely in the past in view of the fact that quantitative removal by adsorption is difficult to achieve even in a laboratory where solution parameters

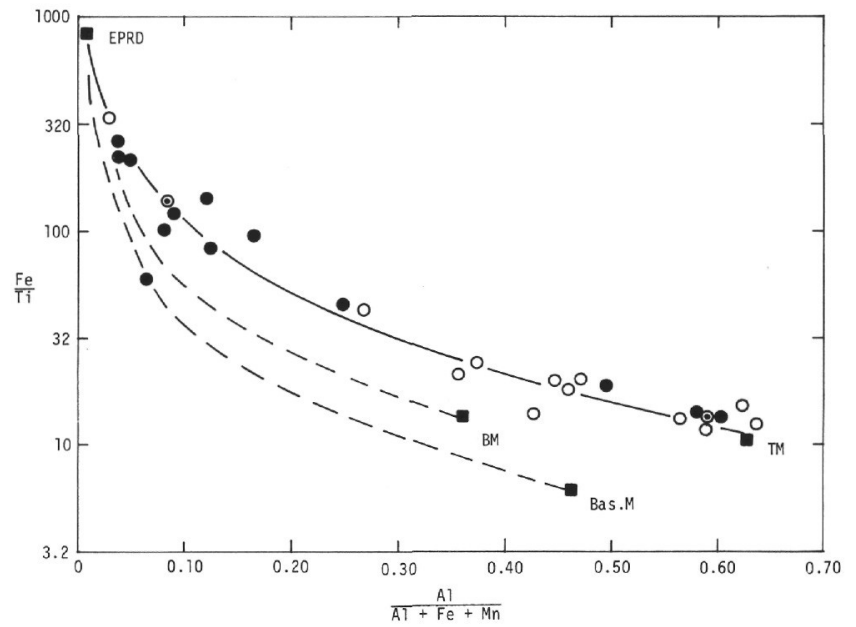

Figure 1. Relationships between $\mathrm{Fe} / \mathrm{Al}$ versus $\mathrm{Al} /(\mathrm{Al}+\mathrm{Fe}$ + Mn). Black dots represent data from this paper (Table 4), rings represent data for sediments from the open Pacific. (Each ring represents the average composition of sediments from one of several areas in the Pacific. Each of these averages is obtained using the average accumulation rates within each area; this procedure avoids the excessive weight that sediments from areas of slow deposition have; see Boström, in press). Double rings represent averages for groups I and II; see Table 7 (data from this paper) and Boström, in press. The black square marked TM represents average terrigenous matter (see Table 8), and black square marked Bas.M represents average oceanic, tholeiitic basalt (see Manson, 1968). Black square BM represents average marine biological matter (see Table 8). It is obvious that mixing of average $T M$ with average oceanic BM or average marine BM cannot explain much of the compositional variations in East Pacific sediments that concerns $\mathrm{Al}, \mathrm{Fe}, \mathrm{Mn}$, and Ti. Mixing of average terrigenous matter with the extreme type of East Pacific Rise sediments such as GS 7202-35, black square marked EPRD, or Risepac 68, 69 (Boström and Peterson, 1969), on the other hand, gives an excellent fit between observed and predicted (solid curve) relations. This shows that almost all deep-sea sediments can be considered as mixtures of EPRD and TM with regard to the major constituents $\mathrm{Al}, \mathrm{Fe}, \mathrm{Mn}$, and Ti. The figure further demonstrates that mixtures of basaltic matter with EPR sediments are virtually absent. These results are in excellent agreement with previously observed $\mathrm{Fel}$ $\mathrm{Ti}-\mathrm{Al} /(\mathrm{Al}+\mathrm{Fe}+\mathrm{Mn}$ ) relations (Boström, 1970; Boström et al., 1972b; 1973b). Furthermore, note that the EPRmatter has a fairly constant composition with a very high $\mathrm{Fe} / \mathrm{Al}$ ratio; otherwise, it would be difficult to explain how almost all analyses (out of many hundred) of unconsolidated, nonconcretionary deep-sea sediments with a worldwide distribution fall on or very close to the mixing curve EPRD-TM, both according to data in this paper and in previous reports (Boström, 1970; Boström et al., 1972b). Furthermore, it is of interest to note that as analytical data have improved, the points tend to fall closer to this mixing curve, not scatter farther away from it. 


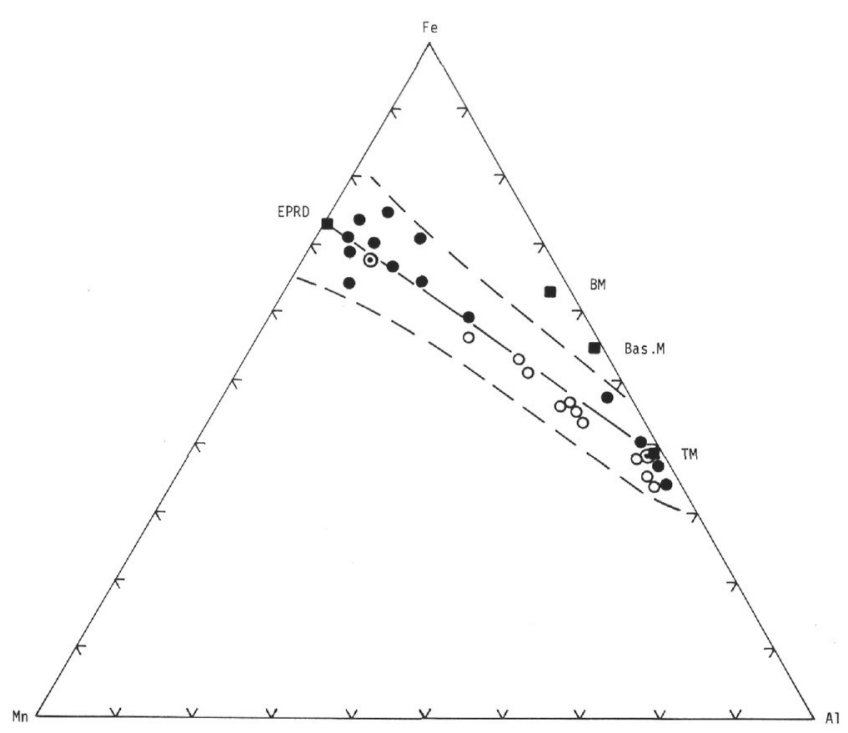

Figure 2. Relationships between $\mathrm{Fe}, \mathrm{Mn}$, and Al in East Pacific and pelagic sediments in general. Symbols are the same as in Figure 1. Note that all sediment averages from this and other works (Boström, in press) fall along the tieline between EPRD and TM, whereas there is no tendency for the points to cluster along the corresponding tielines (not shown) for EPRD-BM or EPRD-Bas.M. This suggests that biological matter and basaltic detritus, etc play a negligible role in the element budgets for $\mathrm{Fe}, \mathrm{Mn}$, and $A l$ in the deep sea. (This does not mean that there are no sediments with heavy admixture of basaltic material, for instance, but that such mixtures are of local significance only, as is well demonstrated by the fact that basaltic components only show up in sediments that accumulate exceedingly slowly, as was shown by Boström et al., 1973b). Note that the relationships in this figure only pertain to unconsolidated, nonconcretionary sediments.

like $p \mathrm{H}$ can be optimized. Note, furthermore, that the model in Figure 4e shows a deficit in $\mathrm{Ni}$ and $\mathrm{Cu}$, that cannot be resolved by the addition of more BM or TM; this will disrupt the major element relationships (see Figure $4 \mathrm{f}$ ). The best explanation, therefore, appears to be that VM is indeed rich in $\mathrm{Cu}$ and $\mathrm{Ni}$; in other words, VM approaches EPRD in composition. It cannot be ruled out at present that the bulk quantity of the EPRD, including its trace content, may have a volcanic source.

\section{General Geochemical Relationships}

A final comment regarding the sediment models above is of interest. Thus, although the quantitative relationships for 10-13 constituents are involved, only two to three input phases are required in the models as demonstrated in Figures $4 \mathrm{a}$ to $4 \mathrm{f}$. Deep-sea sediments far from spreading centers (which are the bulk of all deep-sea sediments) can usually be described with only two inputs, namely BM and TM (Boström et al., 1974b; Boström, in press; Moore et al., in preparation). This suggests that deep-sea sedimentation can, as a firstorder approximation, be considered to be a simpler geochemical process than previously believed. It also implies that mixing of solid phases with fairly constant

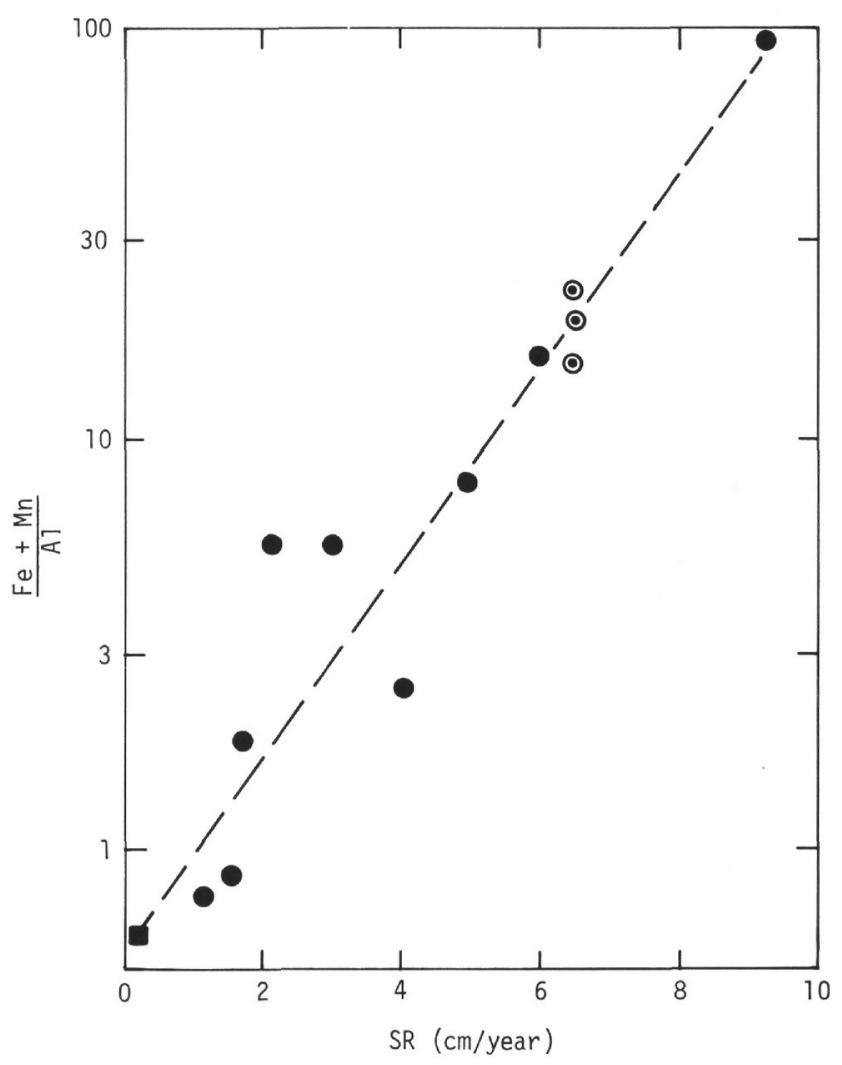

Figure 3. Relationship between crustal spreading rate (SR) and the ratio $\frac{\mathrm{Fe}+\mathrm{Mn}}{\mathrm{Al}}$. Solid circles represent data from

Bostrom (1973); circled dots the basal layers D, G, and $N$ (this work), and squares the TM (Table 8) which is deposited in large quantities in areas of no spreading close to the oceans. The early set of data (solid circles) has a correlation coefficient of 0.93; for all the points in this graph, the correlation has improved to 0.96. Both of these correlation coefficients are well above the value for the $1 \%$ significance level.

compositions plays a much larger role than does transport of dissolved phases for the chemical composition of deep-sea sediments.

In this context it is of particular interest to note the behavior of EPRD in the graphs in Figures 1, 2, and 4 which suggests that the volcanic emanations must hydrolyze almost immediately after they surface on the ocean floor; otherwise one would expect much stronger tendencies for $\mathrm{Fe}, \mathrm{Mn}$, etc to segregate than is observed in the active ridge deposits. This implies that dissolved phases (except in the zone of emanations) play subordinate roles in the formation of deep-sea sediments, possibly with the exception for $\mathrm{Mn}$ and $\mathrm{Ba}$. Other exceptions are, of course, $\mathrm{Na}, \mathrm{K}, \mathrm{Ca}$, and $\mathrm{Sr}$, which are present in seawater in high concentrations.

\section{Special Problems}

\section{Geochemistry of Ba}

The geochemistry of $\mathrm{Ba}$ is perhaps more incompletely known that that of other elements. Association with 


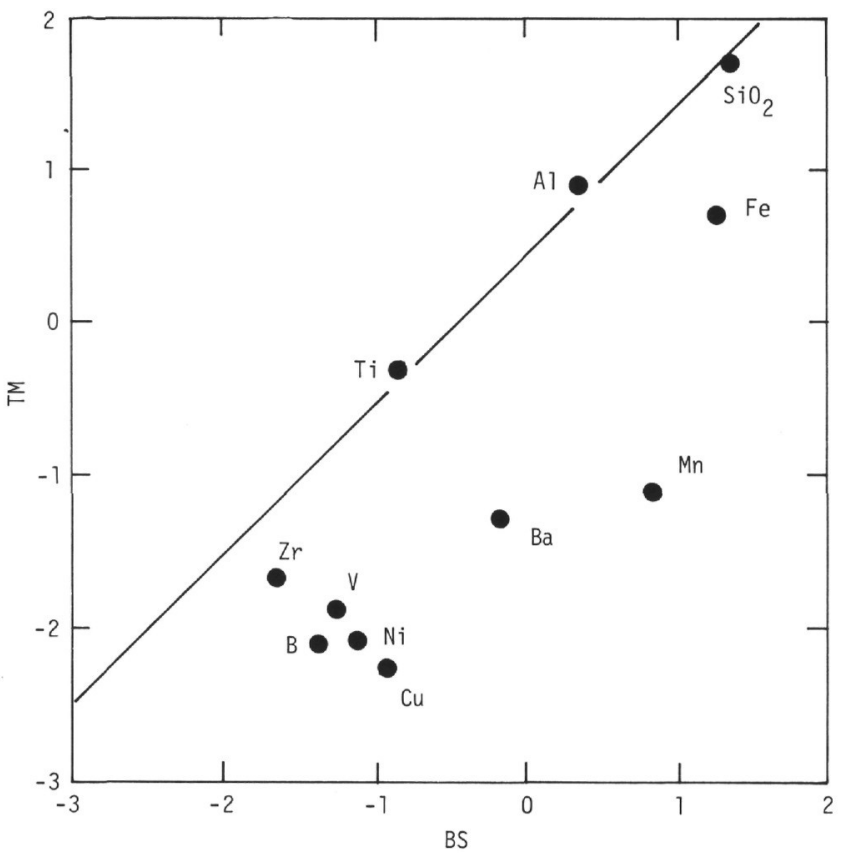

Figure 4a. Geochemical graphs, showing the relationships between the composition of the sediment sources and the sediments in the East Pacific sampled during Leg 34. All values represent the logarithm for the concentration given in \%. Input constituents are from Table 8, in which BM stands for biological matter, TM stands for terrigenous matter, VM stands for volcanic matter (of hypothetical nature), and EPRD stands for East Pacific Rise Deposits as recovered at site GS 7202-35 (see Tables, 6 and 8). Geochemical models of this type have been extensively used and described in Boström et al., 1974a; Boström, in press; Moore et al., in preparation. (a) Relations between TM and average basal sediments (BS) as given in Table 7.

biological processes is obvious, but strong ties with volcanic processes are also evident (Arrhenius and Bonatti, 1965; Bonatti et al., 1972; and Boström et al., 1973a). It is characteristic that modeling for most constituents in the graphs presented in Figure $4 \mathrm{a}$ of this work and in Boström et al. (1974b), is fairly easy. Ba, however, has presented persistent difficulties, that we do not fully understand. It is obvious that our knowledge of how much $\mathrm{Ba}$ is present in BM, TM, and basaltic matter must be wrong, or that $\mathrm{Ba}$ has a migration tendency similar to Mn (Lynn and Bonatti, 1965; Bonatti et al., 1971; Boström and Fischer, 1972).

\section{Geochemistry of Sc and $\mathrm{Zr}$}

The discussion above gives a somewhat erroneous impression of the geochemistry of $\mathrm{Zr}$ and Sc. Thus, Table 7 suggests that the basal layer is richer in these constituents than are the overlying deposits. A more careful study of Table 5 reveals, however, that the lowermost part of the basal section (which generally is the one with the fastest accumulation) shows fairly low concentrations of these elements, as was earlier indicated by studies of recently formed deposits, resting almost

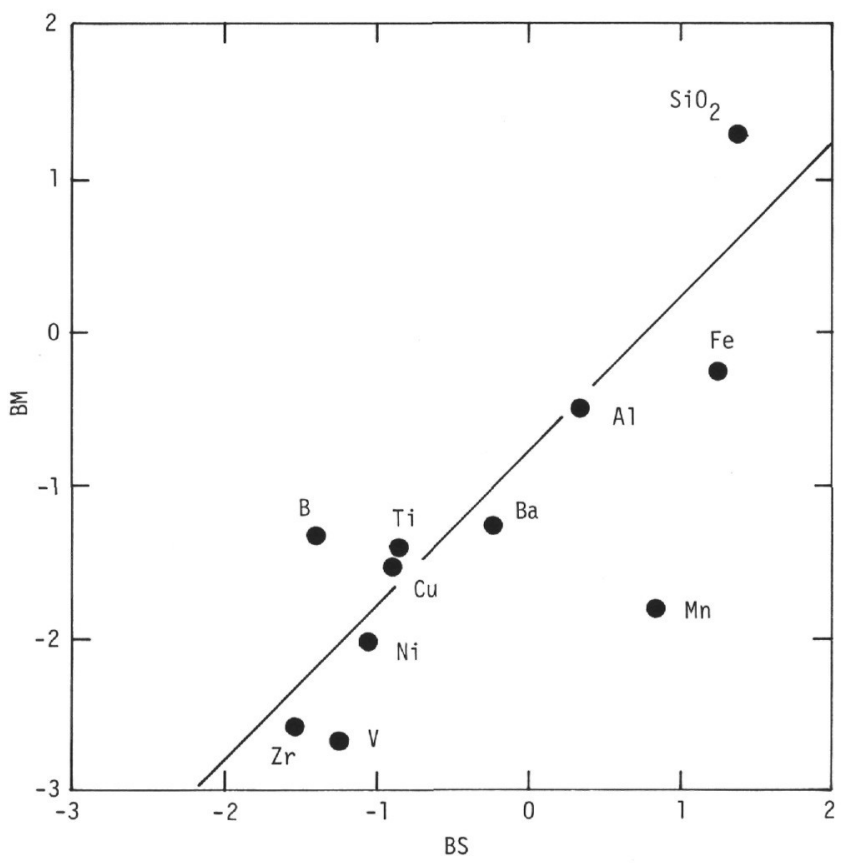

Figure 4b. Relationships between BM and average basal sediments as given in Table 7. In both $(a)$ and $(b)$ the fit is very poor. In these and the following diagrams the diagonal line represents the best fit to the most important hydrolysates $A l$ and Ti; their immobility make them excellent markers of the extent a sediment model can satisfactorily explain the composition of a given sediment. Too little $\mathrm{Mn}, \mathrm{Fe}$, and $\mathrm{V}$ are supplied to models (a) and $(b)$ to explain the origin of the basal sediments in the East Pacific with TM or BM alone.

directly on the basalt substratum. A more proper breakdown of the sediments might therefore have been to divide them in three major layers, of which layer 2, forming far from both spreading centers and from the continents, would show the strongest incorporation of adsorbates and biogenic matter.

\section{Bauer Deep Deposits}

The Bauer Deep deposits have received particularly great attention due to their strange appearance. Boström and Peterson $(1966,1969)$ reported not only very high concentrations of $\mathrm{Fe}$ and $\mathrm{Mn}$, as were known from Revelle's (1944) work, but also very high concentrations of $\mathrm{Cu}, \mathrm{Ni}$, and $\mathrm{Co}$; subsequent studies also have shown very high concentrations of $\mathrm{Zr}, \mathrm{Sc}, \mathrm{Ba}$, and rare earths in these sediments (Boström et al., 1972a, 1973a; Boström, 1973). Similar results as well as very thorough mineralogical studies of the same sediments were reported by Sayles and Bischoff (1973). An extensive discussion of the relationship between heat flow and metallogenesis in the Bauer Deep was presented by Anderson and Halunen (1974).

The findings in this paper do not contradict these experimental results, but perhaps do contradict some earlier conclusions regarding the origin of these sediments. Data in Table 6 suggest that groups A and B at Site 319 (which is representative of present-day sedi- 


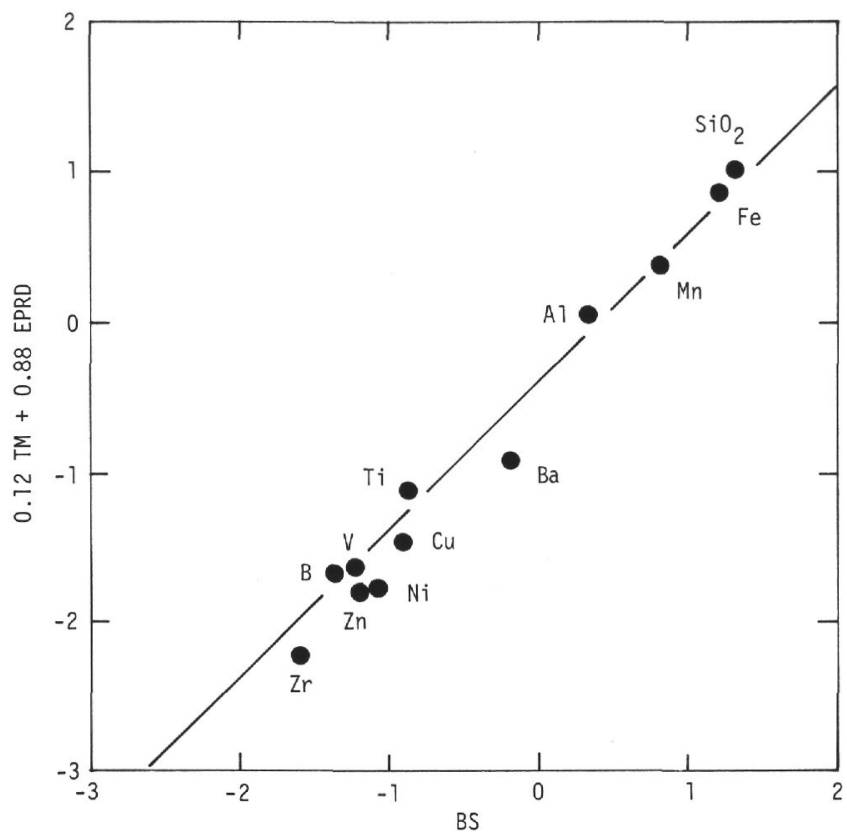

Figure 4c. Relationships between a mixture of $12 \% \mathrm{TM}$ $+88 \%$ EPRD versus average basal sediments. The fit is good, suggesting that not only major constituents (as in Figures 1 and 2) can be explained by mixing of TM and EPRD, but also the abundance of the trace elements.

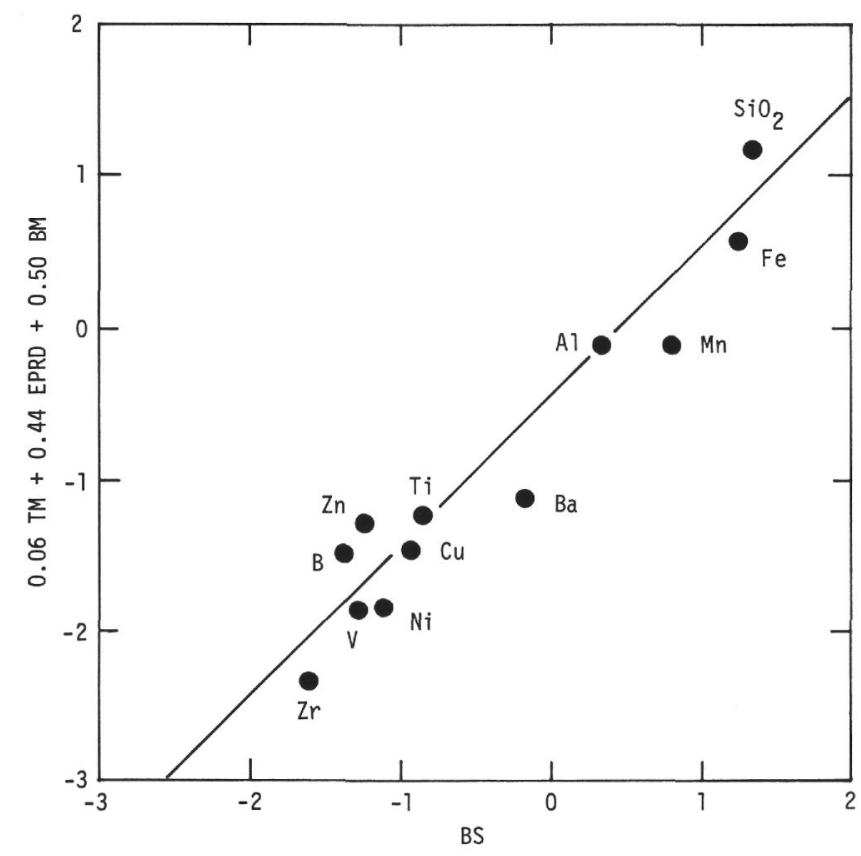

Figure 4d. The model in (c) can be admixed with some BM, but not very much. Already an admixture of $50 \%$ as in this graph gives a poorer fit than in (c). At most, $25 \%$ $B M$ can be admixed without ruining the fit in (c).

mentation conditions in the Bauer Deep) have no unusually high accumulation rates of any constituent; on the contrary, it is a basin with exceedingly sluggish deposition rates at present. This means that adsorption processes, later alterations, hydrothermal "washing" of the sediments, etc will have much time available to leave

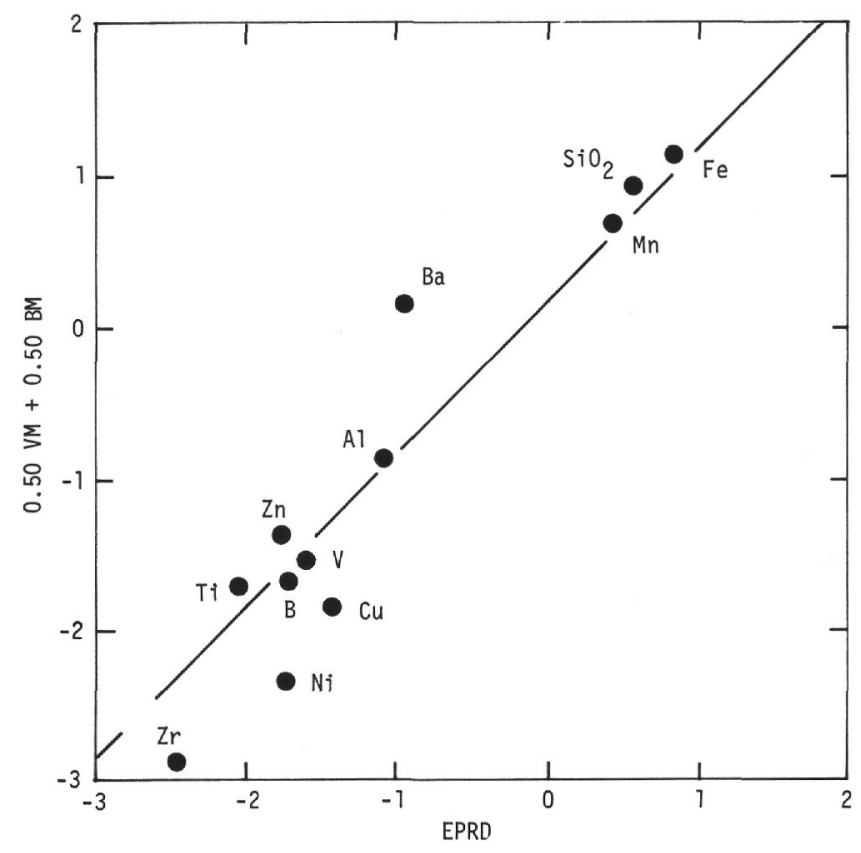

Figure 4e. Model showing how EPRD possibly can be explained as due to mixture of VM and BM in the proportions 1:1. The fit is not very good, however.

their geochemical imprint on these sediments. To use these sediments as particularly useful indicators of what is going on during metallogenesis, as suggested by Anderson and Halunen (1974), is therefore risky. This conclusion is supported by data for groups C and D from Hole 319 (see Tables 5 and 6); the former table shows much lower values for $\mathrm{Ba}, \mathrm{Co}, \mathrm{La}(?), \mathrm{Y}$, and $\mathrm{Zr}$; a decreasing trend may also be present for $\mathrm{Ni}$ and $\mathrm{Sc}$. Note that groups $\mathrm{A}$ to $\mathrm{D}$ fall almost exactly on the mixing curve EPRD-TM in Figure 1.

The conclusion from these findings is that the Bauer Deep is the worst area to study if we want to understand the origin of rapidly forming active ridge deposits. Due to the slow admixture rate of both the typical active ridge sediments as we know them from the crest of the EPR (for instance, GS 7202-35) and of terrigenous matter, the present-day Bauer Deep deposition is exceedingly sensitive to other processes, including trace element or isotope input from other sources. Such studies should be performed on typical active ridge sediments, which accumulate much faster than those in the Bauer Deep.

An additional interesting conclusion can be obtained from the data presented in this paper and in Anderson and Halunen (1974). The very fact that the pronounced heat-flow anomaly in the Bauer Deep (Anderson and Halunen, 1974) has caused such insignificant accumulation rates for the constituents $\mathrm{Fe}, \mathrm{Mn}$, etc., throws severe doubts over the prevailing interpretation that heat flowinduced seawater leaching of the oceanic crust accounts for the origin of the major fraction of the active ridge deposits. This is in good agreement with the findings by Rydell et al. (1974), who conclude that additional sources of a more deep-seated nature are needed to explain the composition of active ridge deposits. 


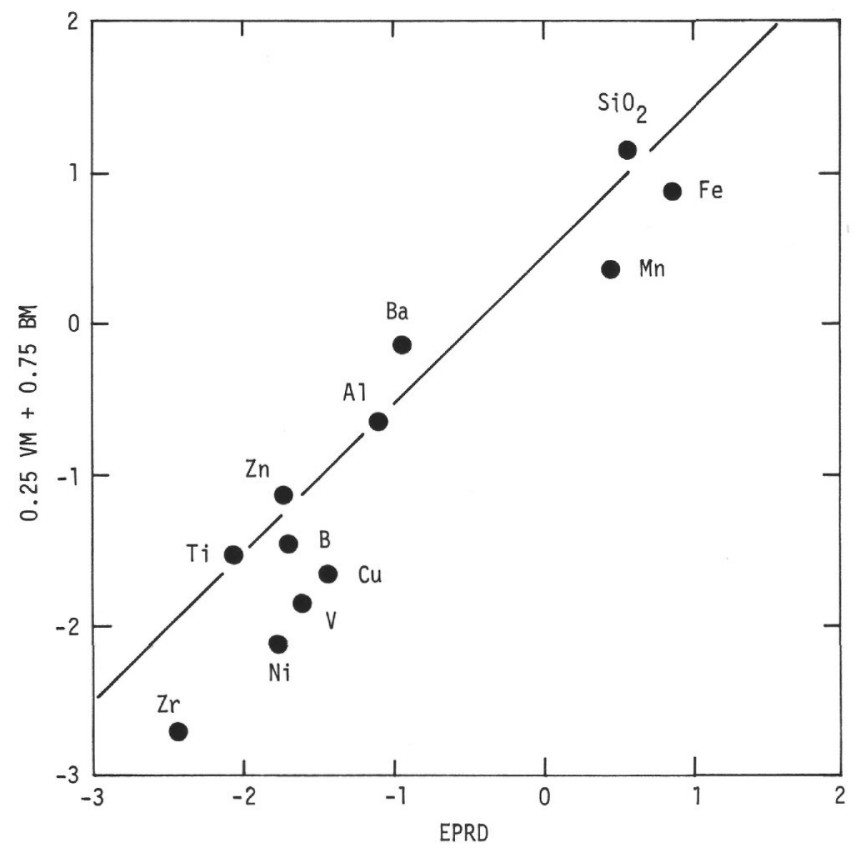

Figure 4f. In the previous model (e) $\mathrm{Cu}, \mathrm{Ni}$, and $\mathrm{Zr}$ values were too low to fit the model well. Additional BM will not improve the situation, as this graph with 1 part $V M$ and 3 parts BM shows; the fit for $F e, M n, B$, and $V$ is lost and the fit for $\mathrm{Cu}$ and Ni has not appreciably improved. This may indicate that a volcanic phase with abundant $\mathrm{Fe}, \mathrm{Mn}, \mathrm{V}$, and $\mathrm{Ba}$ as well as many trace elements such as $\mathrm{Cu}$ and $\mathrm{Ni}$ must be resorted to, that is, the hypothetical VM in Table 8 should approach EPRD in composition.

\section{CONCLUSIONS}

1. Sediments from DSDP Leg 34, which traversed one flank of an active ridge (EPR), show remarkable similarities with those obtained from the spreading ridge in the South Atlantic during DSDP Leg 3. Thus, the basal sediments are rich in $\mathrm{Fe}, \mathrm{Mn}$, and $\mathrm{V}$, and poor in Al. Ti, and Si. The formation of these basal sediments was completed within about $15 \mathrm{~m} . \mathrm{y}$. or less after the generation of the basaltic crust on which the sediments rest.

2. Analyses of chemical data reveal that the basal sediments can be considered to have formed from two components: one a volcanic phase, very similar to the inorganic fraction presently being depositioned on the crest of the EPR (but probably with some admixed biogenous matter); and the second, a terrigenous phase.

3. Accumulation rates for characteristic constituents in this volcanic phase (authigenic $\mathrm{Fe}, \mathrm{Mn}, \mathrm{V}$ ) are very high, often up to $10-100$ times the values found for deposits formed far from spreading centers.

4. Seawater leaching of basaltic rock is an unlikely source for the volcanic emanations deposited in the basal sediments; otherwise one would expect the present-day heat-flow anomalies in the Bauer Deep to be associated with much higher accumulation rates for $\mathrm{Fe}, \mathrm{Mn}$, etc than is now the case. No experimental leaching experiments involving basalt and $\mathrm{NaCl}$ brines have produced the $\mathrm{Al}-\mathrm{Fe}$ separation that is required to explain the composition of active ridge deposits, nor do observations of hot spring waters in basaltic terranes show such separations. These facts contradict the seawater leaching hypothesis.

5. As has been pointed out elsewhere, carbonate-rich emanations of deep-seated origin are the most likely source for the anomalously Fe-Mn-rich active ridge deposits, as well as for much of their content of $\mathrm{Ba}, \mathrm{P}$, and U. Such a source could also explain why active ridge deposits are so persistently poor in $\mathrm{Si}, \mathrm{Al}, \mathrm{Ti}$, and $\mathrm{Th}$ compared to other deep-sea sediments.

6. Studies of sediment models suggest that the volcanic emanations hydrolyze immediately after they surface, and that they subsequently, in a particulate form, are admixed with terrigenous and biogenous matter in various proportions. This implies that outside the emanation zone, dissolved matter and solutions play but a small role in the origin of deep-sea sediments, an interpretation that is supported by the data in Figures 1, 2 , and 4 .

\section{ACKNOWLEDGMENTS}

This research was supported by the National Science Foundation Grant NSF-GX-40428. This is a contribution from the Rosenstiel School of Marine and Atmospheric Sciences and from the Department of Economic Geology, University of Lulea.

\section{REFERENCES}

Anderson, R.N. and Halunen, A.J., 1974. Implications of heat flow for metallogenesis in the Bauer Deep: Nature v. 251, p. 473-475.

Arrhenius, G.O.S. and Bonatti, E. 1965. Neptunism and volcanism in the ocean. In Sears, M. (Ed.), Progress in oceanography, Volume 3: Washington (Am. Assoc. Adv. Sci.), p. 7-22.

Barth, T.F.W., 1952. Theoretical petrology: New York (John Wiley \& Sons).

Berggren, W.A., 1969. Rates of evolution in some Cenozoic planktonic foraminifera: Micropaleontology, v. 15, p. 351365.

Bonatti, E., Fisher, D.E., Joensuu, O., and Rydell, H.S., 1971. Postdepositional mobility of some transition elements, phosphorous, uranium, and thorium in deep-sea sediments: Geochim. Cosmochim. Acta, v. 35, p. 189-201.

Bonatti, E., Fisher, D.E., Joensuu, O., Rydell, H., and Beyth, M.. 1972. Iron-manganese-barium deposit from the Northern Afar rift (Ethiopia): Econ. Geol., v. 67, p. 717730.

Boström, K., 1967. The problem of excess manganese in pelagic sediments. In Abelson, P.M. (Ed.), Researches in geochemistry, Volume 2: New York (John Wiley \& Sons), p. 421-452.

.1970. Submarine volcanism as a source for iron: Earth Planet. Sci. Lett., v. 9, p. 348-354.

1973. The origin and fate of ferromanganoan active ridge sediments: Acta Univ. Stockholmiensis, Stockholm Contrib. Geol. v. 27, p. 149-243.

, in press. Particulate and dissolved matter as sources for pelagic sediments: Acta Univ. Stockholmiensis, Stockholm Contrib. Geol.

Boström, K. and Fisher, D.E., 1972. Lateral fluctuations in pelagic sedimentation during the Pleistocene glaciations: Boreas, v. 1, p. 275-288. 
Boström, K., and Peterson, M.N.A., 1966. Precipitates from hydrothermal exhalations on the East Pacific Rise: Econ Geol., v. 61, p. 1258-1265. 1969. Origin of aluminum-poor ferromanganoan sediments in areas of high heat flow on the East Pacific Rise: Marine Geol., v. 7, p. 427-447.

Boström, K., Farquharson, B., and Eyl, W., 1972a. Submarine hot springs as a source of active ridge sediments: Chem. Geol., v. 10, p. 189-203.

Boström, K., Joensuu, O., Valdés, S., and Riera, M., 1972 b. Geochemical history of South Atlantic Ocean sediments since late Cretaceous: Marine Geol., v. 12, p. 85-121.

Boström, K., Joensuu, O., Moore, C., Boström, B., Dalziel, M., and Horowitz, A., 1973a. Geochemistry of Ba in pelagic sediments: Lithos, v. 6, p. 159-174.

Boström, K., Kraemer, T., and Gartner, S., 1973b. Provenance and accumulation rates of opaline silica, $\mathrm{Al}, \mathrm{Ti}$, $\mathrm{Fe}, \mathrm{Mn}, \mathrm{Cu}, \mathrm{Ni}$ and $\mathrm{Co}$ in Pacific pelagic sediments: Chem Geol., v. 11, p. 123-148.

Boström, K., Joensuu, O., Kraemer, T., Rydell, H., Valdés, S., Gartner, S., and Taylor, G., 1974a. New finds of exhalative deposits on the East Pacific Rise: Geol. Fören. Stockh. Förhandl., v. 96, p. 53-60.

Boström, K., Joensuu, O., and Brohm, I., 1974b. Planktonits chemical composition and its significance as a source of pelagic sediments. Chem. Geol., v. 14, p. 255-271.

Corliss, J.B., 1971. The origin of metal-bearing submarine hydrothermal solutions: J. Geophys. Res., v. 76, p. 81288138.

Green, H.W., II, 1972. A CO 2 charged asthenosphere: Nature, Phys. Sci., v. 238. p. 2-5.

Hays, J.D., Cook, H., Jenkins, G., Orr, W., Goll,.R., Cook, F., Milow, D., and Fuller, J., 1972. An interpretation of the geologic history of the Eastern Equatorial Pacific from the drilling results of Glomar Challenger, Leg 9. In Hays, J.D. et al., 1972. Initial Reports of the Deep Sea Drilling Proj- ect, Volume 9: Washington (U.S. Government Printing Office), p. 909-931.

Herron, E.M., 1972. Sea-floor spreading and Cenozoic history of the East Central Pacific: Geol. Soc. Am. Bull., v. 83, p. 1671-1692.

Krauskopf, K.B., 1967. Introduction to geochemistry: New York (McGraw-Hill).

Landergren, S., 1964. On the geochemistry of deep sea sediments. Rept. Swedish Deep Sea Exped., v. 5, p. 1-154.

Lynn, D.C. and Bonatti, E., 1965. Mobility of manganese in diagenesis of deep-sea sediments: Marine Geol., v. 3, p. 457-474.

Manson, V., 1968. Geochemistry of basaltic rocks: major elements. In Hess, H.H. and Poldervaart, A., (Eds.), Basalts: New York (John Wiley \& Sons), v. 1, p. 215-269.

Moore, C. and Boström, K., in preparation. Lower marine organisms: their geochemistry and role as a source for trace elements in deep sea deposits.

Revelle, R.R., 1944. Marine bottom samples collected in the Pacific by the Carnegie on its seventh cruise: Washington (Carnegie Inst. Wash.), Publ. 556.

Rydell, H., Kraemer, T., Boström, K., and Joensuu, O., 1974. Postdepositional injections of U-rich solutions into East Pacific Rise sediments: Marine Geol., v. 17, p. 151-164.

Sayles, F.L. and Bischoff, J.L., 1973. Ferromanganoan sediments in the Equatorial East Pacific: Earth Planet. Sci. Lett., v. 19, p. 330-336.

Sillitoe, R.H., 1972. Relations of metal provinces in Western America to subduction of oceanic lithosphere: Geol. Soc. Am. Bull., v. 83, p. 813-818.

Turekian, K.K., 1968. Deep-sea deposition of barium, cobalt and silver: Geochim. Cosmochim. Acta, v. 32, p. 603-612.

van Andel, Tj.H., Corliss, J.B., and Bowen, V.T., 1967. The intersection between the Mid-Atlantic Ridge and the Vema Fracture Zone in the North Atlantic: J. Mar. Res.; Sears Found. Mar. Res., v. 25, p. 343-351. 